ARTICLE

Received 31 May 2016 | Accepted 3 Aug 2016 | Published 20 Sep 2016

DOI: $10.1038 /$ ncomms12815

OPEN

\title{
A dendritic disinhibitory circuit mechanism for pathway-specific gating
}

Guangyu Robert Yang ${ }^{1}$, John D. Murray ${ }^{1,2} \&$ Xiao-Jing Wang ${ }^{1,3}$

While reading a book in a noisy café, how does your brain 'gate in' visual information while filtering out auditory stimuli? Here we propose a mechanism for such flexible routing of information flow in a complex brain network (pathway-specific gating), tested using a network model of pyramidal neurons and three classes of interneurons with connection probabilities constrained by data. We find that if inputs from different pathways cluster on a pyramidal neuron dendrite, a pathway can be gated-on by a disinhibitory circuit motif. The branch-specific disinhibition can be achieved despite dense interneuronal connectivity, even with random connections. Moreover, clustering of input pathways on dendrites can naturally emerge through synaptic plasticity regulated by dendritic inhibition. This gating mechanism in a neural circuit is further demonstrated by performing a context-dependent decision-making task. The model suggests that cognitive flexibility engages top-down signalling of behavioural rule or context that targets specific classes of inhibitory neurons.

\footnotetext{
${ }^{1}$ Center for Neural Science, New York University, 4 Washington Place, New York, New York 10003, USA. ${ }^{2}$ Department of Psychiatry, Yale University School of Medicine, New Haven, Connecticut 06511, USA. ${ }^{3}$ NYU-ECNU Institute of Brain and Cognitive Science, NYU Shanghai, Shanghai 200122, China.

Correspondence and requests for materials should be addressed to X.-J.W. (email: xjwang@nyu.edu).
} 
$\mathrm{D}$ istinct classes of inhibitory interneurons form cell-typespecific connections among themselves and with pyramidal neurons in the cortex ${ }^{1,2}$. Interneurons expressing parvalbumin (PV) specifically target the perisomatic area of pyramidal neurons. Interneurons expressing somatostatin (SOM) specifically target thin basal and apical tuft dendrites of pyramidal neurons $s^{3,4}$. Interneurons expressing vasoactive intestinal peptide (VIP) avoid pyramidal neurons and specifically target SOM neurons ${ }^{5}$. Long-range connections from cortical $^{6,7}$ or subcortical $^{8}$ areas can activate VIP neurons, which in turn suppress SOM neurons, and disinhibit pyramidal dendrites. Such dendritic disinhibitory circuit is proposed to gate excitatory inputs targeting pyramidal dendrites ${ }^{9-11}$ (Fig. 1a).

Insofar as any cortical area receives inputs from tens of other areas and projects to many other areas, information flow across the complex cortical circuit needs to be flexibly gated (or routed) according to behavioural demands. Broadly speaking, there are three types of gating in terms of specificity. First, all inputs into a cortical area may be uniformly modulated up or down. Recent research in mice demonstrated that such gating involves the disinhibitory motif mediated by VIP and SOM interneurons ${ }^{6,8,12-15}$. These studies generally found that VIP neurons are activated, and SOM neurons are inactivated, in response to changes in the animals' behavioural states, such as when mice receive reinforcement ${ }^{14}$, or start active whisking ${ }^{6,15}$ or running 8 . The reported state change-related activity responses can be remarkably homogeneous across the local population of the same class of interneurons ${ }^{12,13}$

Second, gating may involve selective information about a particular stimulus attribute or spatial location (for instance, in visual search or selective attention ${ }^{7}$ ). Whether SOM or VIP neurons are endowed with the required selectivity remains insufficiently known. In sensory cortex, SOM neurons exhibit greater selectivity to stimulus features (such as orientation of a visual stimulus) than PV neurons ${ }^{16}$. Furthermore, in motor cortex, SOM neurons have been shown to be highly heterogeneous and remarkably selective for forward versus backward movements ${ }^{17}$.

Third, for a given task, neurons in a cortical area may need to 'gate in' inputs from one of the afferent pathways and 'gate out' other afferent pathways ${ }^{18,19}$, which we call 'pathway-specific gating'. For instance, imagine yourself sitting in a noisy café and trying to focus on your book. Your associational language areas receive converging inputs from both auditory and visual pathways. Opening the gate for the visual pathway while closing the gate for the auditory pathway allows you to focus on reading (Fig. 1b). In the classic Stroop task, the subject is shown a coloured word, and is asked to either name the colour or read the word. One possible solution to this task is for a decisionmaking area to locally open its gate for the deliberate pathway (colour-naming) while closing its gate for the more automatic pathway (word-reading).

Using computational models, we propose that the dendritic disinhibitory circuit can instantiate pathway-specific gating. Each of the many branches of a pyramidal dendrite processes its inputs quasi-independently ${ }^{20}$ and nonlinearly ${ }^{21}$. Feedforward and feedback pathways target different regions (for example, basal or apical tuft) of dendritic trees of pyramidal neurons ${ }^{22}$. We hypothesize that excitatory inputs from different pathways can cluster onto parts of dendrites of pyramidal neurons, which we term 'branch-specific' even though inputs from a particular pathway may target multiple branches. This hypothesis is supported by mounting evidence for synaptic clustering on dendritic branches ${ }^{23-25}$. A pathway can presumably be 'gated-on' by specifically disinhibiting the branches targeted by this pathway (Fig. 1c), that is, by a disinhibition pattern aligned with the excitation. This branch-specific disinhibition is motivated by findings showing that synaptic inhibition from SOM neurons can act very locally on dendrites, even controlling individual excitatory synapse by targeting the spine ${ }^{4}$ or the pre-synaptic terminal $^{26}$. In this work, we developed a network model with thousands of pyramidal neurons and hundreds of interneurons for each (VIP, SOM and PV) type, and show that pathwayspecific gating can be accomplished by the disinhibitory motif, even though the connectivity from SOM neurons to pyramidal neurons is dense: each SOM neuron on average targets $>60 \%$ of neighbouring pyramidal neurons $(<200 \mu \mathrm{m})^{27}$.

We first characterized how branch-specific disinhibition can efficiently gate excitatory inputs onto pyramidal dendrites. We then investigated the plausibility of branch-specific disinhibition in a dendritic disinhibitory circuit model constrained by experimentally measured single-neuron physiology and circuit connectivity. We found that, although SOM-topyramidal connectivity is dense at the level of neurons, at the level of dendrites it is sufficiently sparse to support branchspecific disinhibition, and therefore pathway-specific gating, given
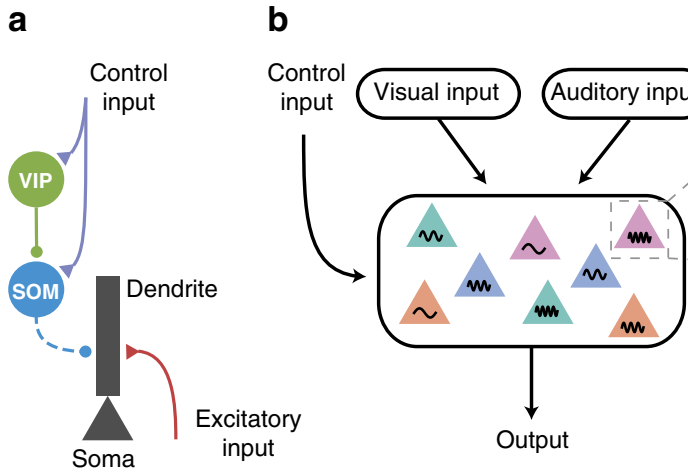

C Visual pathway gate open

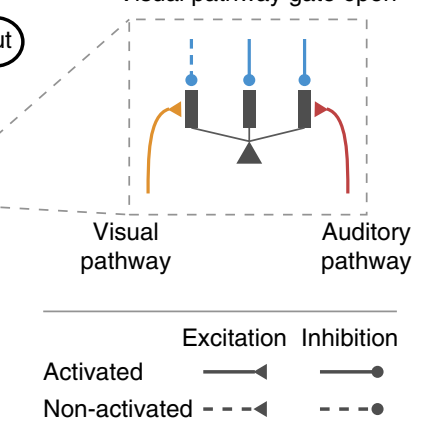

Figure 1 | Dendritic disinhibitory circuit as a mechanism for pathway-specific gating. (a) Subcellular microcircuit motif for gating through dendritic disinhibition. Dendrites of pyramidal neurons are inhibited by SOM interneurons, which are themselves inhibited by VIP interneurons. A control input (representing a context or a task rule) targeting VIP interneurons (and potentially SOM neurons) can thereby disinhibit pyramidal neuron dendrites, opening the gate for excitatory inputs targeting these dendrites. (b) Circuit configuration for pathway-specific gating. Pyramidal neurons receive converging inputs from multiple pathways, for example, visual and auditory. Single neurons in these areas are selective to multiple stimulus features, indicated here by colour and frequency. The processing of each pathway is regulated by the control input. (c) Inputs from different pathways target distinct subsets of dendrites of these pyramidal neurons. A pathway can be gated-on by specifically disinhibiting the dendrites that it targets, corresponding to an alignment between excitation and disinhibition. Disinhibition is represented by dashed lines. 
that SOM neurons can be selectively controlled. We then showed control inputs targeting both VIP and SOM neurons can selectively suppress SOM neurons as needed. Notably, we drew these conclusions under some 'worst-case' assumptions to our model such as random interneuronal connectivity. Using a calcium-based synaptic plasticity model, we found that disinhibitory regulation of plasticity can give rise to an appropriate alignment of excitation and disinhibition, which is required for pathway-specific gating in our scheme. Finally, we demonstrated the functionality of this mechanism in a circuit model performing an example context-dependent decision-making task ${ }^{28}$.

Our results suggest that, in addition to the proposal that SOM neurons provide a 'blanket of inhibition'29, they can indeed subserve pathway-specific gating. This work argues that top-down behavioural control may involve rule signals targeting specific interneuron types rather than, or in addition to, pyramidal neurons, and that the disinhibitory motif could play a major role in synaptic plasticity.

\section{Results}

Pathway-specific gating with dendritic disinhibition. To study dendritic disinhibition, we first built a simplified neuron model

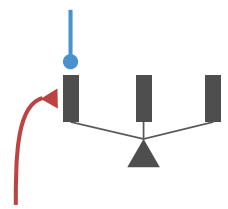

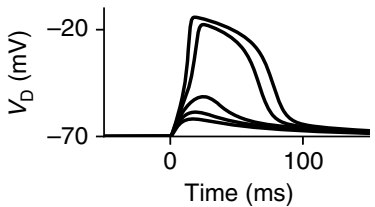

C

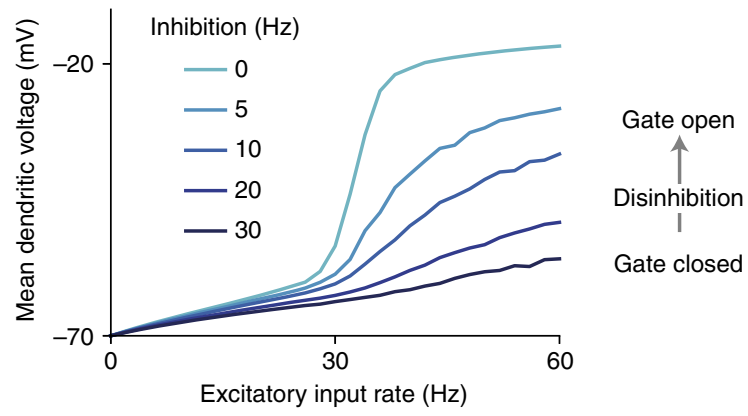

d

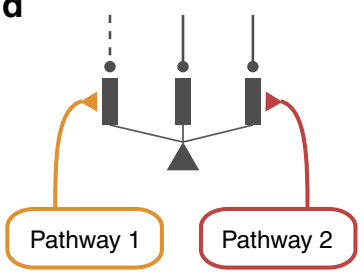

f

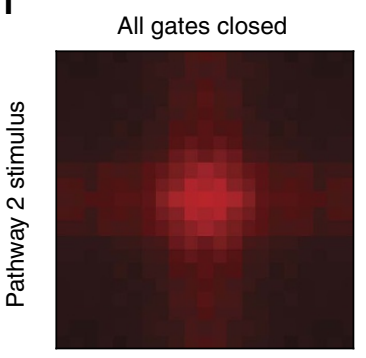

Pathway 1 stimulus e

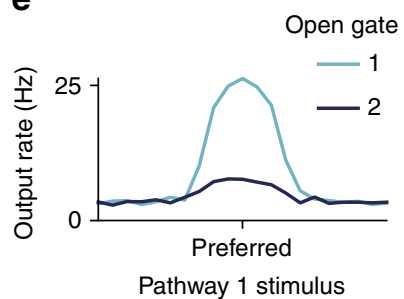

g

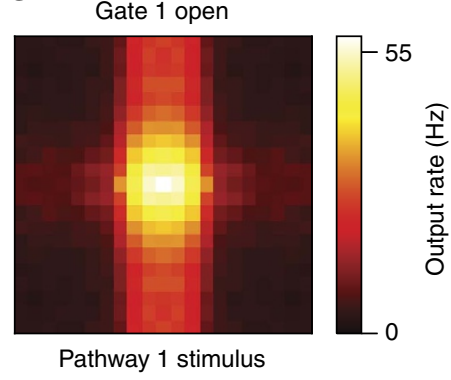

with a reduced morphology (Fig. 2a; Supplementary Fig. 1). It comprises one spiking somatic compartment and multiple dendritic compartments, which are electrically coupled to the soma but otherwise independent of each other. The somatic and dendritic compartments have no spatial extent themselves. This choice of morphology is inspired by previous studies showing that different dendritic branches can integrate their local input independently from one another ${ }^{20}$.

A prominent feature of active processing in thin dendritic branches is their ability to produce NMDA ( $N$-methyl-Daspartate) plateau potentials ${ }^{30}$, also called NMDA spikes. The NMDA plateau potential is a regenerative event in which the membrane potential increases nonlinearly and sometimes sharply with the NMDAR ( $N$-methyl-D-aspartate receptor) input, due to the release of voltage-dependent magnesium block of NMDARs. The reduced neuron model can exhibit NMDA plateau potential in dendrites (Fig. 2b), in line with simulations of morphologically reconstructed neuron models (Supplementary Fig. 1). The mean dendritic voltage in response to a Poisson spike train input is a sigmoidal function of the input rate, due to the NMDA plateau potential (light blue curve in Fig. 2c).

The NMDA plateau potential can be prevented by applying a moderate synaptic inhibition, mediated by $\mathrm{GABA}_{\mathrm{A}} \mathrm{Rs}$ ( $\gamma$-aminobutyric acid receptors), to the same dendrite (dark blue curve in Fig. 2c). Inhibition is particularly effective in controlling this dendritic nonlinearity when excitatory inputs are mediated by NMDARs with experimentally observed saturation, in stark contrast to AMPARs ( $\alpha$-amino-3-hydroxy-5-methyl4-isoxazolepropionic acid receptors; Supplementary Fig. 1) or NMDARs without saturation (Supplementary Fig. 2). Inhibitory input also linearizes the relationship between mean dendritic voltage and excitatory input rate (Fig. 2c), due to stochastic transitions into or out of NMDA plateau potential induced by low-rate inhibition (Supplementary Fig. 2). Therefore, excitatory inputs to a dendritic branch can be efficiently gated by inhibition $^{31}$

We now consider multiple pathways of inputs targeting distinct sets of dendrites. In the default condition, all dendritic

Figure 2 | Context-dependent gating of specific pathways. (a) A reduced compartmental neuron with a somatic compartment connected to multiple, otherwise independent, dendritic compartments (only three shown).

(b) Excitatory inputs can generate a local, regenerative NMDA plateau potential in the dendrite. As number of activated synapses increased, there is a sharp nonlinear increase in the evoked dendritic membrane depolarization $\left(V_{D}\right)$. (c) Disinhibition of the targeted branch opens the gate for the excitatory input. (d) A pyramidal neuron receives converging inputs from multiple pathways carrying different stimulus features, giving it selectivity to a preferred stimulus for each feature dimension. Each input pathway targets separate dendrites, which are disinhibited correspondingly in each context by top-down control inputs (not modelled here). (e) Tuning curve for input pathway 1 , when only this pathway is activated. The input pathway encodes a stimulus feature, for example, motion direction, with a bell-shaped tuning curve for the input. The preferred feature value corresponds to higher input firing rate. When gate 1 is open by disinhibiting the dendrites targeted by input pathway 1 , the neuron exhibits strong tuning (light blue). When gate 2 is instead open, the neuron exhibits weak tuning for the feature (dark blue). The amount of inhibition reduced for a disinhibited dendrite, that is, the disinhibition level, is $30 \mathrm{~Hz}$. (f,g) Twodimensional tuning curves when both pathways are activated. $(\mathbf{f})$ In the default context, no dendrites are disinhibited and both pathways are gatedoff. The neuron exhibits weak responses regardless of the stimulus features. (g) When gate 1 is open by disinhibiting branches targeted by pathway 1 , the response of this neuron is dominated by tuning to the pathway 1 stimulus, although pathway 2 has a residual impact. 
branches receive a high baseline inhibition from dendritetargeting SOM neurons 6,15 , closing gates for all pathways. Disinhibiting the branches targeted by one pathway can selectively open the gate for this pathway while keeping the gates closed for other pathways (Fig. 2d). When a gate is open, the neuron's output firing rate transmits the stimulus selectivity of the corresponding input pathway most effectively (Fig. 2e).

When two excitatory pathways are activated simultaneously, we can plot the neuron's response to stimulus variables of both pathways, that is, the two-dimensional tuning curve (Fig. 2f,g). In the default condition when all gates are closed, there is little response to either pathway (Fig. 2f). By specifically disinhibiting the branches targeted by pathway 1 , we can open the gate for pathway 1 . With gate 1 opened, the neuron is primarily selective to pathway 1 stimuli (Fig. $2 \mathrm{~g}$ ). The remaining impact of pathway 2 stimuli is due to the fact that the impact of excitatory inputs can never be fully counteracted by dendritic inhibition.

The gating mechanism worsens when a fraction of excitatory input is mediated by AMPARs, but improves when a fraction of inhibitory input is mediated by $\mathrm{GABA}_{\mathrm{B}}$ Rs (Supplementary Fig. 3). Under in vivo conditions, the relative contribution of AMPAR-mediated inputs is likely quite low, as a result of a lower glutamate affinity and a stronger desensitization ${ }^{21}$. For parsimony, in the following sections, excitatory synaptic inputs are mediated only by NMDARs, which are critical to the nonlinear dendritic computations, and inhibitory inputs are mediated only by $\mathrm{GABA}_{\mathrm{A}}$ Rs.

Performance of gating in pyramidal neurons. Which circuit properties determine the effectiveness of pathway-specific gating in our model? A neuron responds to its optimal stimulus from an input pathway with (baseline-corrected) firing rate $\left(r_{\text {on }}\right)$ when the pathway is gated-on, and ( $\left.r_{\text {off }}\right)$ when the pathway is gated-off, which could be readily measured experimentally. The gating selectivity is then quantified,

$$
\text { Gating selectivity }=\frac{r_{\mathrm{on}}-r_{\mathrm{off}}}{r_{\mathrm{on}}+r_{\mathrm{off}}}
$$

which ranges from 0 (no gating) to 1 (perfect gating). We developed a multi-compartmental rate model $^{20}$ that greatly improves the efficiency of the circuit model simulation. The rate model is fitted to quantitatively reproduce the activity of the spiking neuron model (Supplementary Fig. 4; see Supplementary Information for details).

We first tested how gating selectivity depends on our assumption of branch-specific disinhibition in a single-neuron setting. Here we assume an alignment of excitation and disinhibition patterns, which can be achieved through synaptic plasticity as shown later. Each excitatory pathway targets $N_{\text {disinh }}$ randomly chosen dendrites, out of $N_{\text {dend }}$ total dendrites, and this pathway is gated-on by specifically disinhibiting these same $N_{\text {disinh }}$ dendrites (Fig. 3a). Due to the random-independent selection of targeted dendrites for each pathway, inputs from two different pathways often overlap.

We found that gating selectivity depends critically on the sparseness of the disinhibition (Fig. 3b), defined as the proportion of targeted/disinhibited dendrites $N_{\text {disinh }} / N_{\text {dend }}$. Gating selectivity improves when disinhibition patterns are sparsened, because the proportion of dendrites that receive overlapping inputs is reduced. We can approximate the limit of $N_{\text {disinh }} / N_{\text {dend }} \rightarrow 0$ with non-overlapping disinhibition pattern (diamonds in Fig. 3b,c). In this case, the gating selectivity is highest but below 1 , due to the remaining impact of inputs targeting inhibited dendrites, and is therefore modulated by the level of disinhibition (Fig. 3c).

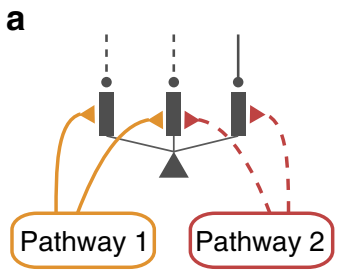

Gate 1 open Firing rate: $r_{\text {on }}$

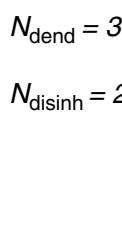

Gating selectivity: $\left(r_{\text {on }}-r_{\text {off }}\right) /\left(r_{\text {on }}+r_{\text {off }}\right)$

b

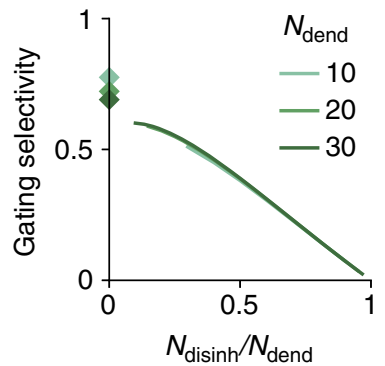

C

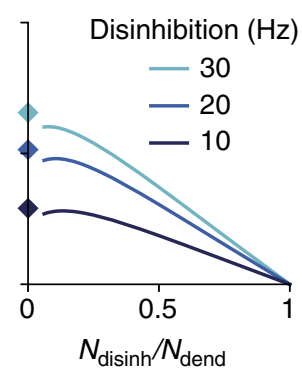

Figure 3 | Characterization of gating selectivity in pyramidal neurons.

(a) Schematic of gating, presenting pathway 1 input when gate 1 is opened (left) or gate 2 is opened (right). There are $N_{\text {dend }}$ available dendrites in total. Each input pathway targets $N_{\text {disinh }}$ dendrites. To gate a pathway on, these exact $N_{\text {disinh }}$ dendrites are disinhibited, creating an aligned pattern of disinhibition. Each pathway selects dendrites randomly and independently from other pathways, which can result in overlap of the excitationdisinhibition patterns across pathways. When $N_{\text {disinh }}$ is large, projections from different pathways are more likely to overlap. The neuron's firing rate is $r_{\text {on }}$ and $r_{\text {off }}$ in response to the preferred stimulus of the gated-on (left) and gated-off (right) pathway, respectively. The gating selectivity is defined as $\left(r_{\text {on }}-r_{\text {off }}\right) /\left(r_{\text {on }}+r_{\text {off }}\right)$, which is 1 for perfect gating and 0 for no gating. (b) Gating selectivity increases as excitation/disinhibition patterns become sparser, that is, with a smaller proportion of targeted and disinhibited dendrites for a pathway $\left(N_{\text {disinh }} / N_{\text {dend }}\right)$. Diamonds mark the case of nonoverlapping excitatory projections, corresponding to the limit of maximal sparseness. (c) Gating selectivity is higher with stronger disinhibition, for all sparseness levels.

Pathway-specific gating with SOM neurons. We have shown that a key determinant of gating performance is the sparseness of innervation patterns onto the dendritic tree. Yet, the connectivity from SOM interneurons to pyramidal neurons is dense ${ }^{27}$. Is it possible for the proposed gating mechanism to function in a cortical microcircuit limited by the dense interneuronal connectivity? To address this issue, we built an interneuronal circuit model, containing hundreds of VIP and SOM interneurons and thousands of pyramidal neurons. We considered 'worst-case' conditions in which interneuronal connectivity is completely random (as our gating mechanism can be facilitated by structured connectivity). Surprisingly, we found that relatively high gating performance is achievable under these conditions. We analysed gating in this circuit in two steps: first, assuming SOM neurons are context-selective, we characterized how the SOM-pyramidal subcircuit can support high gating selectivity. Second, we characterized how SOM neurons can become contextselective in the VIP-SOM-pyramidal circuit.

First, we built a simplified model of a SOM-pyramidal subcircuit (Fig. 4a), which corresponds roughly to a cortical L2/3 column $(400 \times 400 \mu \mathrm{m})$. The model contains $N_{\text {pyr }}(\approx 3,000)$ multi-compartmental pyramidal neurons, each with $N_{\text {dend }}(\approx 30)$ dendrites and $N_{\text {SOM }}(\approx 160)$ SOM neurons (Supplementary 
a

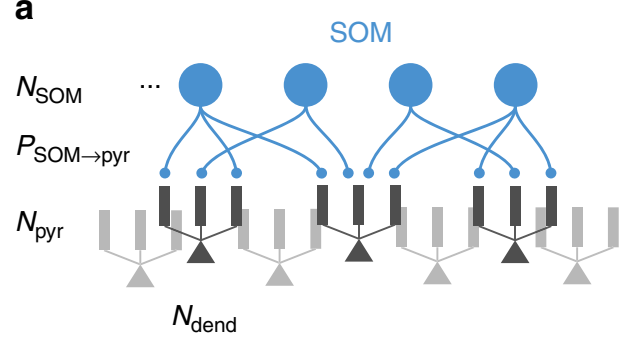

C

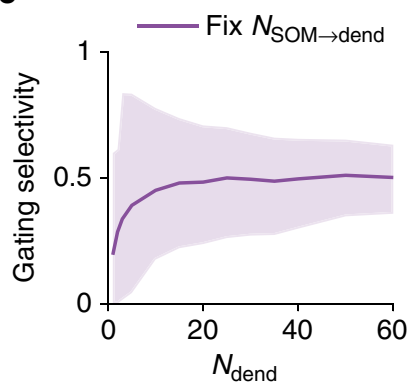

e

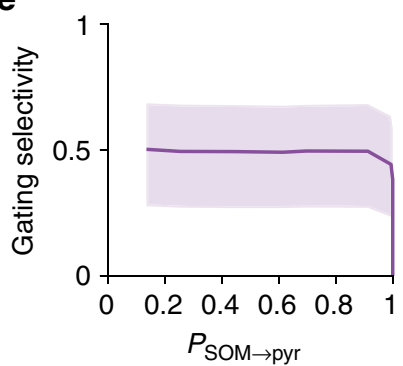

b

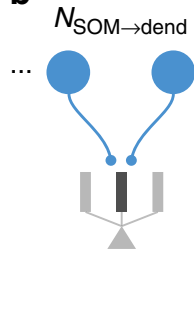

d

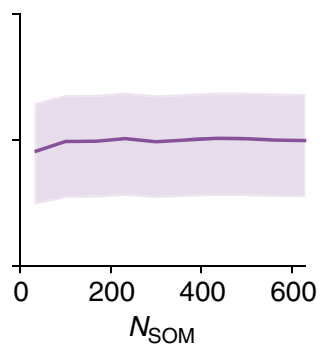

f

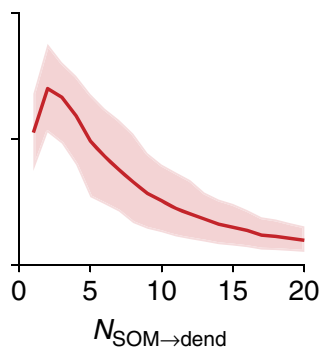

Figure 4 | Gating selectivity as functions of SOM-pyramidal circuit parameters. (a) A simplified model for a cortical column of SOM and pyramidal neurons. We only modelled the SOM-to-pyramidal connections. The model is subject to experimentally measured constraints of the following parameters: number of SOM neurons $\left(N_{S O M}\right)$, connection probability from SOM to pyramidal neurons $\left(P_{\mathrm{SOM} \rightarrow \mathrm{pyr}}\right)$, and the number of dendrites on each pyramidal neuron $\left(N_{\text {dend }}\right)$. We consider the 'worst-case' scenario that the SOM-to-dendrite connections are random. Finally, we assume for now that control input for each pathway suppresses a random subset of SOM neurons. The different contrasts used are for illustration purpose only. (b) A critical parameter for the SOM-topyramidal circuit is the number of SOM neurons targeting each dendrite $\left(N_{\mathrm{SOM}} \rightarrow\right.$ dend $)$. This parameter can be calculated using other experimentally measured parameters under the assumption of random connectivity,

$N_{\mathrm{SOM} \rightarrow \text { dend }}=N_{\mathrm{SOM}} \cdot\left[1-\left(1-P_{\mathrm{SOM} \rightarrow \mathrm{pyr}}\right)^{1 / N_{\text {dend }}}\right]$. (c-e) Gating selectivity only weakly depends on $N_{\text {dend }}(\mathbf{c}), N_{\mathrm{SOM}}(\mathbf{d})$ and $P_{\mathrm{SOM} \rightarrow \text { pyr }}(\mathbf{e})$ if $N_{\mathrm{SOM} \rightarrow \text { dend }}$ is kept constant by co-varying another parameter. The plotted curve marks the mean and the shaded region marks the bottom $10 \%$ to top $10 \%$ of the neuronal population. (f) Gating selectivity is high when each dendrite is targeted by a few SOM neurons. Given experimental measurements of $P_{\text {SOM } \rightarrow \text { pyr }} \approx 0.6, N_{\text {dend }} \approx 30$ and $N_{\text {SOM }} \approx 160$, we obtained $N_{\text {SOM } \rightarrow \text { dend }} \approx 5$, leading to relatively high gating selectivity $\sim 0.5$. Total strength of inhibition onto each pyramidal dendrite is always kept constant when varying parameters.

Table 1). Here we analyse the dependence of gating selectivity on the connectivity from SOM to pyramidal neurons. We consider worst-case conditions in which these connections are random, subject to the SOM-to-pyramidal connection probability of $P_{\mathrm{SOM} \rightarrow \mathrm{pyr}}(\approx 0.6)$. Assuming that a SOM neuron chooses to target each pyramidal dendrite independently with a

SOM-to-dendrite connection probability of $P_{\mathrm{SOM} \rightarrow \text { dend }}$ then we have

$$
\begin{aligned}
P_{\mathrm{SOM} \rightarrow \mathrm{dend}} & =1-\left(1-P_{\mathrm{SOM} \rightarrow \mathrm{pyr}}\right)^{1 / N_{\mathrm{dend}}} \\
& \approx P_{\mathrm{SOM} \rightarrow \mathrm{pyr}} / N_{\mathrm{dend}}, \text { for small } P_{\mathrm{SOM} \rightarrow \mathrm{pyr}} .
\end{aligned}
$$

Under this assumption, a SOM neuron on average targets $N_{\text {dend }} \cdot P_{\text {SOM } \rightarrow \text { dend }} / P_{\mathrm{SOM} \rightarrow \text { pyr }} \approx 1.5$ dendrites of a pyramidal neuron given that the two are connected. Each SOM-dendrite connection can correspond to multiple (3-5) clustered synapses $^{32}$. So each SOM neuron can make on average 5-8 synapses onto a pyramidal neuron. The connection probability between two neurons is higher at closer proximity ${ }^{27}$, leading to a even higher number of contacts.

In a default state, SOM neurons fire at a relatively high baseline rate around $10 \mathrm{~Hz}$ (refs 6,15), closing the gates to all inputs. To open the gate for pathway 1, a randomly chosen subset (50\%) of SOM neurons is suppressed, resulting in a pattern of disinhibition across dendrites. Again, we assume the excitatory input pattern of pathway 1 is aligned with the corresponding disinhibition pattern. Notably, disinhibition patterns for different pathways generally overlap due to the random selection of SOM neurons and the random connectivity. This overlap can be reduced with either structured connections or inhibitory plasticity.

Under the above assumptions, the circuit achieves a mean gating selectivity $\sim 0.5$, equivalent to $r_{\text {on }} \approx 3 r_{\text {off. }}$. We found that the impact of these circuit parameters is determined by one critical parameter: the number of SOM neurons targeting each dendrite $N_{\mathrm{SOM} \rightarrow \text { dend }}=N_{\mathrm{SOM}} \cdot P_{\mathrm{SOM} \rightarrow \text { dend }} \approx 5$ (Fig. $4 \mathrm{~b}$, see also Supplementary Note 1). When we vary parameters while keeping $N_{\text {SOM } \rightarrow \text { dend }}$ fixed, the gating selectivity remains largely constant (Fig. 4c-e). We found that gating selectivity is highest when $N_{\text {SOM } \rightarrow \text { dend }}$ is small (Fig. 4f), and decreases as we increase $N_{\mathrm{SOM} \rightarrow \text { dend. }}$. Because the overall strength of inhibition has a simple scaling effect on the gating selectivity (Fig. 3c), we keep it fixed when varying other parameters.

Each dendrite should more appropriately be interpreted as an independent computational unit. When inhibitory connections control individual excitatory connection through pre-synaptic receptors ${ }^{26}$ or by targeting spines ${ }^{4}$, the independent unit would be single excitatory synapses. This leads to a lower effective value of $N_{\mathrm{SOM} \rightarrow \text { dend }}$, then a higher gating selectivity.

Pathway-specific gating with SOM and VIP neurons. Having analysed the SOM-pyramidal connectivity, we next examined how SOM neurons can be context-selective, and characterized the gating selectivity in a circuit model containing VIP, SOM and pyramidal neurons. On top of the previous SOM-pyramidal subcircuit, We added $N_{\text {VIP }}$ VIP neurons that only target SOM neurons ${ }^{5}$. Here we assume VIP neurons target all SOM neurons with connection probability $P_{\mathrm{VIP} \rightarrow \mathrm{SOM}}$. Broadly speaking, we found two scenarios in which SOM neurons can be suppressed selectively based on the context, depending on the targets of the top-down or locally generated control inputs (Fig. 5).

In the first scenario, control inputs target VIP neurons solely (Fig. 5a). In this intuitive scenario, control inputs excite VIP neurons, which in turn inhibit SOM neurons thereby disinhibiting pyramidal dendrites. Gating selectivity is high only if a small proportion of VIP neurons is targeted by control (Fig. 5b), indicating that VIP neurons must be context-selective, and VIPto-SOM connections need to be sparse (Fig. 5c). VIP-to-SOM connectivity could possibly be effectively sparse on the scale of a cortical column, since the axonal arbour of VIP neurons are rather spatially restricted ${ }^{33}$. When varying parameters, we kept 
a

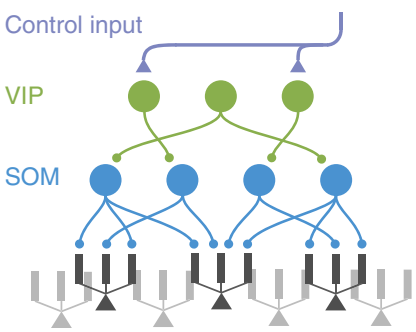

d

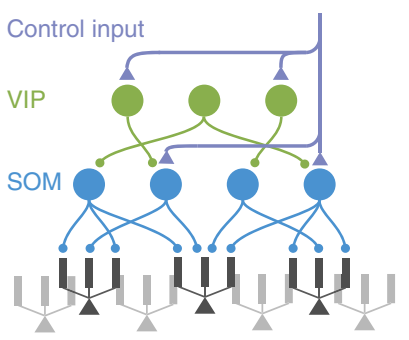

b

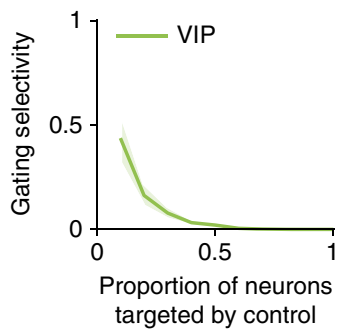

e

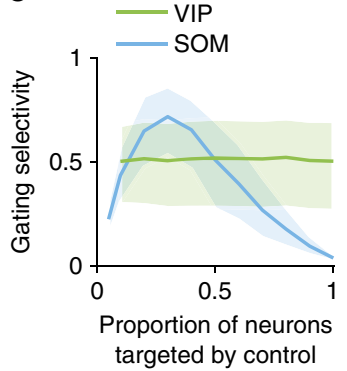

C

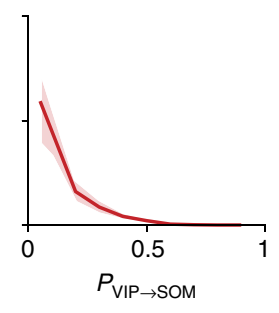

f

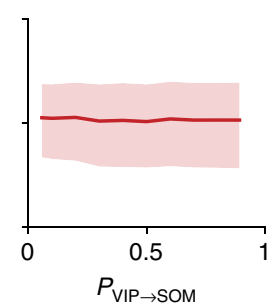

Figure 5 | Two possible schemes of control for the interneuronal circuit. We built a simplified circuit model containing VIP, SOM and pyramidal neurons. (a-c) Control signals target only VIP neurons. (a) In this scheme, for each pathway, control inputs target a random subset of VIP neurons. And the connection probability from VIP to SOM neurons is $P_{\mathrm{VIP} \rightarrow \mathrm{SOM}}(\mathbf{b}, \mathbf{c})$ Good gating selectivity is only achieved when a small subset of VIP neurons is targeted by control inputs (b), and when the VIP-SOM connections are sparse (c). (d-f) Control signals target both VIP and SOM neurons. (d) In this scheme, we assume that for each pathway control inputs target a random subset of VIP and SOM neurons. (e) Gating selectivity depends on the proportion of SOM (blue) but not VIP (green) neurons targeted by control input. (f) Gating selectivity does not depend on $P_{\mathrm{VIP} \rightarrow \text { soM. }}$. Curves and shaded regions are as in Fig. 4.

fixed the overall baseline inhibition received by each SOM neuron and the overall strength of control inputs.

In the second scenario, excitatory control inputs target both VIP and SOM neurons (Fig. 5d). If the VIP-to-SOM connectivity is dense, then VIP neurons activated by control inputs will provide nearly uniform inhibition across all SOM neurons (Supplementary Fig. 5). However, SOM neurons can receive selective excitation if the control inputs only directly target a randomly chosen subset of SOM neurons. If the inhibition is on average stronger, then the overall effect is a selective suppression of SOM neurons (Supplementary Fig. 5). As a result, gating selectivity no longer depends on the proportion of VIP neurons targeted by control inputs, but does depend on the proportion of SOM neurons targeted (Fig. 5e). Therefore, SOM neurons need to be context-selective, but VIP neurons need not be. Similarly, gating selectivity does not depend on the connection probability from VIP to SOM neurons, $P_{\mathrm{VIP} \rightarrow \text { SOM }}$ (Fig. 5f).

In summary, to achieve branch-specific disinhibition, control inputs targeting interneurons have to be selective. Notably, the level of specificity required for the control inputs depends strongly on the neurons they target. When targeting only VIP neurons, the control inputs have to be highly selective (Fig. 5a,b). However, when control inputs target both VIP and SOM neurons, high gating selectivity can be achieved in a much broader range of parameters, reducing the level of specificity required (Fig. 5d,e).

Pathway-specific gating with SOM, VIP and PV neurons. PV neurons receive inhibition from themselves and SOM neurons, and project to perisomatic areas of pyramidal neurons ${ }^{1}$. Suppression of SOM neurons therefore also leads to the disinhibition of PV neurons and an increase of somatic inhibition onto pyramidal neurons. We included PV neurons into our interneuronal circuit model (Fig. 6a) and found that this
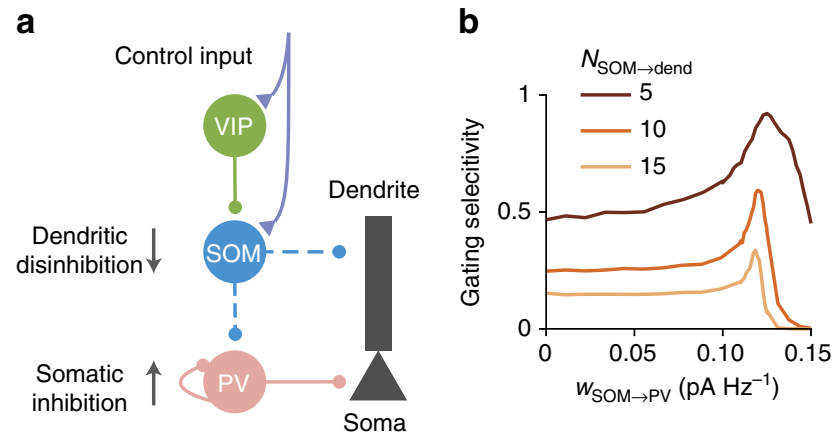

Figure 6 | Somatic inhibition improves gating selectivity. (a) PV neurons project to the somatic areas of pyramidal neurons, and are inhibited by SOM neurons and themselves. Suppression of SOM neurons causes disinhibition of PV neurons, therefore an increase in somatic inhibition onto pyramidal neurons. (b) A moderate increase in somatic inhibition always improves gating selectivity. We included PV neurons and their corresponding connections in the model of Fig. $5 \mathrm{~d}$. Gating selectivity increases as a function of the SOM-to-PV connection weights ( $W_{\mathrm{SOM} \rightarrow \mathrm{PV}}$ ) in a wide range (see Supplementary Note 1 for a proof). However, when gating selectivity is low without PV neurons (light curve), the peak of this increase is lower and the slope is sharper. Gating selectivity starts to decrease when the SOM-to-PV connection, therefore the somatic inhibition, is too strong that the responses of many pyramidal neurons are completely suppressed.

inclusion and the consequent increase in somatic inhibition strictly improve gating selectivity in a wide range of parameters (Fig. 6b). Since the SOM-to-PV and PV-to-pyramidal neuron connections are dense ${ }^{29}$, a selective pattern of SOM suppression will result in an elevated somatic inhibition that is almost uniform across pyramidal neurons (Supplementary Fig. 6). Furthermore, 
we proved that a uniform increase in somatic inhibition will always improve gating selectivity, except when the somatic inhibition is unreasonably strong (Supplementary Note 2).

For an intuitive explanation, consider a linear input-output function in the soma. Gating selectivity is based on the relative difference between the pyramidal neuron responses when the gate is open $\left(r_{\text {on }}\right)$ and when the gate is closed $\left(r_{\text {off }}\right)$. Providing an equal amount of somatic inhibition in these two conditions is equivalent to subtracting both values by the same constant, which will enhance the relative difference.

Learning pathway-specific gating. A critical feature of our scheme is the alignment between excitation and disinhibition patterns (Fig. 1c): pyramidal dendrites targeted by an excitatory input pathway are also disinhibited when the gate is open for that pathway. Dendritic disinhibition can regulate synaptic plasticity $^{34,35}$. We hypothesized that such an alignment can naturally arise as a result of the regulated plasticity. To test this hypothesis, we first established a realistic calcium-based plasticity model for

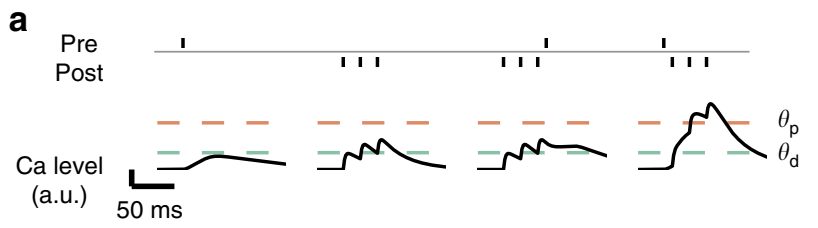

b

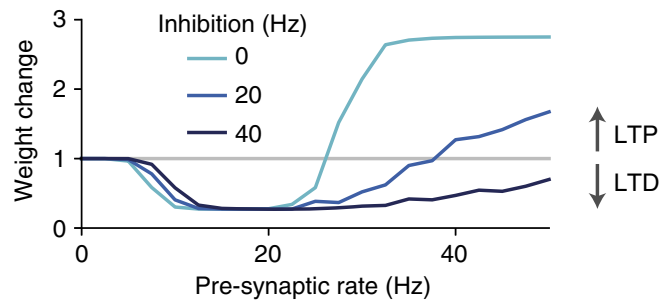

C

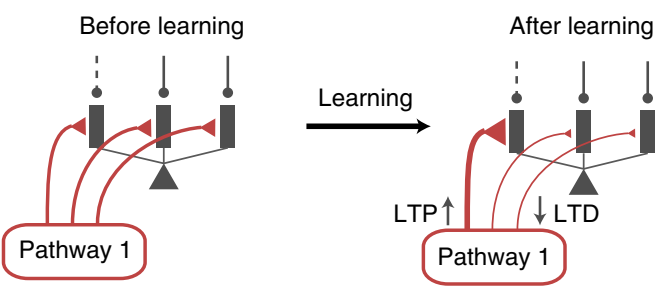

d
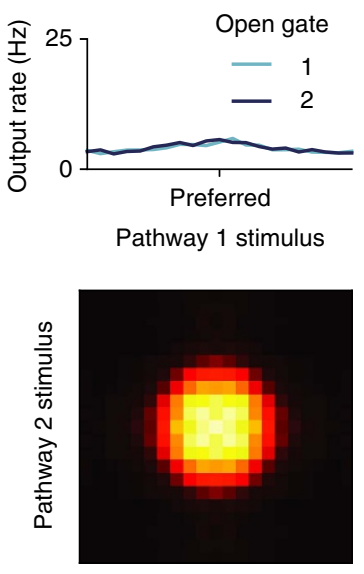

Pathway 1 stimulus e
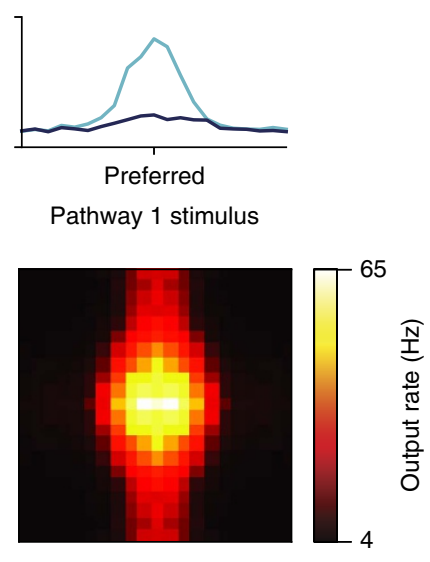

Pathway 1 stimulus dendrites in our reduced spiking neuron model. Pre- and postsynaptic spikes induce calcium transients in dendrites, which determine the synaptic weight changes ${ }^{36}$ (Fig. 7a). We fitted parameters of the model to capture experimental data ${ }^{37}$ (Supplementary Fig. 7). Our model also quantitatively predicts findings that were not used in the fitting.

The calcium-based plasticity model allows us to naturally study the effects of dendritic disinhibition on synaptic plasticity and their functional implications. Again, we assume that pre- and post-synaptic firings are Poisson spike trains with specified rates. We found that dendritic inhibition can shift the plasticity from potentiation to depression, even when the pre-synaptic excitatory input rate and the post-synaptic firing rate are both kept constant (Fig. 7b), consistent with the previous modelling findings ${ }^{35}$. We note that plasticity models based solely on pre- and post-synaptic neuronal firing would not predict the inhibitory modulation of synaptic plasticity.

We then tested whether disinhibitory regulation of plasticity can support the development of excitation-disinhibition alignment, as needed for pathway-specific gating (Fig. 7c). Importantly, the strength of disinhibition is realistic, similar to those used throughout this paper. Initially, excitatory synapses from each pathway are uniformly distributed across the dendritic branches of single neurons. Different excitatory pathways are then activated one at a time. Whenever a pathway is presented, a particular subset of dendrites is disinhibited, while the rest of the dendrites remain inhibited. Through calcium-based excitatory plasticity, the activated excitatory synapses targeting the disinhibited dendrites become strengthened, whereas those targeting the inhibited dendrites become weakened. Synapses not activated remain the same regardless of the inhibition level (Fig. 7b). After learning, the alignment of excitation and disinhibition patterns support pathway-specific gating (Fig. 7d,e; compare with Fig. 2e),

Figure 7 | Learning to gate specific pathways. (a) Model schematic. Pre- and post-synaptic spikes both induce calcium influx. The overall synaptic weight change is determined by the amount of time the calcium level spends above thresholds for depression $\left(\theta_{\mathrm{d}}\right)$ and potentiation $\left(\theta_{\mathrm{p}}\right)^{36}$. The model is fitted to the experimental data, and is able to quantitatively predict results not used in the fitting. (b) Dendritic inhibition makes potentiation harder to induce. With background-level inhibition (light blue), synaptic weight change shows three regimes as a function of excitatory input rate: no change for low rate, depression for medium rate and potentiation for high rate. With a medium level of inhibition (dark blue), potentiation requires a higher excitatory input rate. With relatively strong inhibition (black), potentiation becomes impossible within a reasonable range of excitatory input rates. The post-synaptic rate is fixed at $10 \mathrm{~Hz}$. (c) Learning paradigm. Left: excitatory synapses from each pathway are initialized uniformly across dendrites. When pathway 1 is activated, specific branches of the neuron are disinhibited (dashed line), that is, gate 1 is open. During learning, only one pathway is activated at a time. Right: after learning, activated excitatory synapses onto the disinhibited branches are strengthened, while activated synapses onto inhibited branches are weakened, resulting in an alignment of excitation and disinhibition patterns. Synaptic weights of non-activated synapses remain unchanged (not shown). (d) Response properties of the neuron before learning. Top: tuning curve of the neuron when only pathway 1 is presented. The neuron shows no preference to the gate opened prior to learning. Bottom: twodimensional tuning curve of the neuron when both pathways are simultaneously presented and gate 1 is open. See Fig. 2 for the definition of the tuning curves. (e) Response properties of the neuron after learning. Top: the neuron shows strong tuning to pathway 1 input when gate 1 is open. Bottom: when both pathways are presented, the neuron's response is primarily driven by pathway 1 stimulus, although pathway 2 stimulus also affects the neuron's firing. 
a

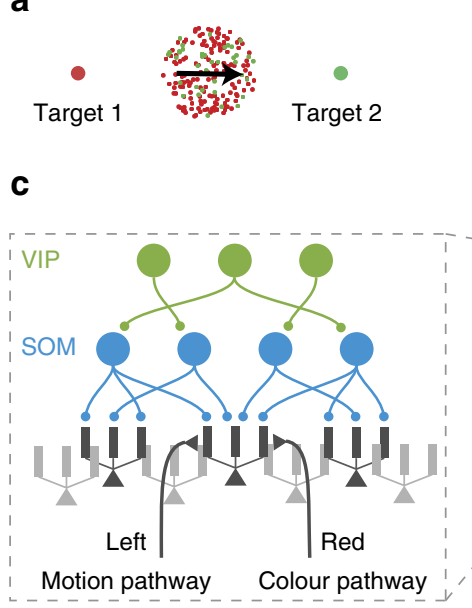

b

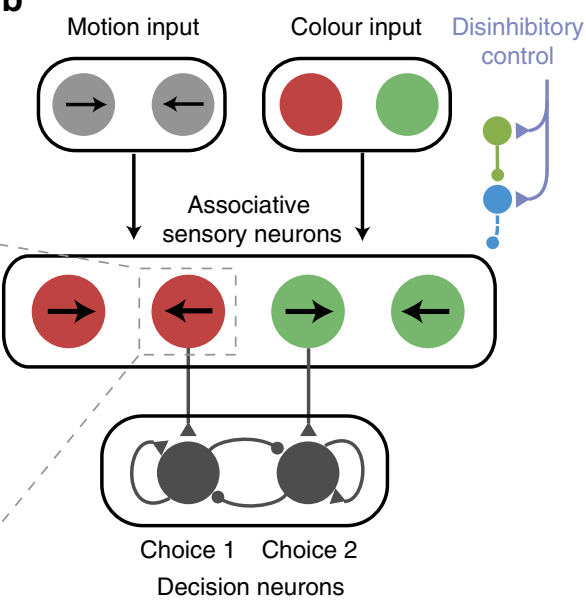

d

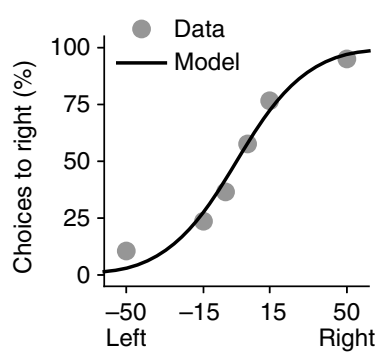

Motion coherence e

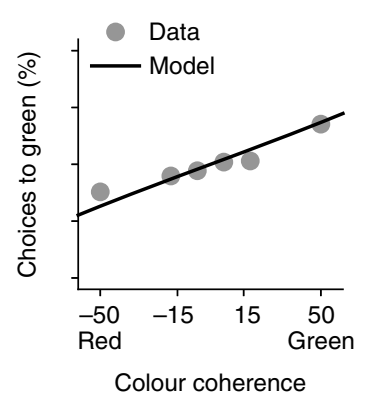

f

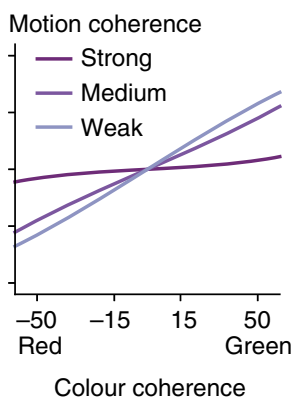

Figure 8 | Pathway-specific gating in an example context-dependent decision-making task. (a) A flexible decision-making task. Depending on the context, the subject's behavioural response should be based on either the colour or the motion direction of the stimulus. (b) The circuit model scheme. Motion and colour pathways target associative sensory neurons, which are subject to context-dependent disinhibitory control. Neurons preferring colour and motion evidence for the same target project to the corresponding neural pool in the decision-making circuit. (c) Associative sensory neurons receive converging inputs from both motion and colour pathways, and are controlled by the dendrite-targeting interneuronal circuit. (d-f) Fit and prediction of behavioural performance. Behavioural performance in the motion context as a function of motion coherence (d) and colour coherence (e) for a monkey (dots), and the model's fit (line). Experimental data are extracted from ref. 28. The model can capture the behavioural performance of a monkey. (f) In the model, the impact of the irrelevant pathway (colour) is strongest when the relevant pathway signal is weak (with low motion coherence).

with a gating selectivity $\sim 0.7$. These findings show that a key aspect of the gating architecture, namely, the alignment of excitation and disinhibition patterns, can emerge naturally from the interaction between excitatory synaptic plasticity and contextdependent disinhibition.

Modelling a flexible behaviour with pathway-specific gating. How is gating at the neural level related to gating at the behavioural level? Is moderate gating selectivity (for example, $\sim 0.5$ as above) sufficient to explain performances in flexible cognitive tasks? To address these issues, we applied our model to a contextdependent decision-making task ${ }^{28}$. In this task, the behavioural response should be based on either the motion direction or the colour of a random-dots motion stimulus, depending on the context cued by a rule signal (Fig. 8a).

We built a stylized neural circuit model to implement this task using pathway-specific gating through dendritic disinhibition (Fig. 8b). The local circuit comprises a sensory network and a decision network. The sensory network contains pyramidal neurons that receive convergent sensory inputs from both motion and colour pathways, and they group into four pools according to their selectivities to colour and motion evidence. The dendrites of pyramidal neurons are controlled by the VIP-SOM interneuronal circuit described above (Figs 8c,5d). A subset of pyramidal neurons with high gating selectivity projects to the decision network. Pyramidal neurons representing colour and motion evidence for the same target project to the corresponding decision neural pool. The decision network, as modelled previously ${ }^{38}$, is a strongly recurrent network that generates a winner-take-all decision based on its inputs.

We fitted the performance of the model to a monkey's psychometric behavioural data from ref. 28, using three free parameters in the model, namely, the proportion of sensory neurons that project to the decision network, and the overall connection strengths from the input pathways to the sensory network and from the sensory network to the decision network. By fitting these three parameters, we obtained a quantitative match of the empirical psychometric performance, as a function of relevant (Fig. 8d) and irrelevant (Fig. 8e) features. Our model shows that the impact of the irrelevant information should be stronger when the relevant information is more ambiguous (with lower motion coherence, for instance; Fig. 8f). Although at its default parameters the interneuronal circuit model can show similar task performance as the empirical data, we found that it can no longer fit the empirical performance if we significantly degrade the neural gating selectivity (Supplementary Fig. 8). This simulation therefore serves as a proof of principle to demonstrate 
the potential of dendritic disinhibition as a mechanism for pathway gating, and as a link to assess the utility of neural gating selectivity in terms of flexible behavioural performance.

\section{Discussion}

A canonical cortical microcircuit motif specialized for disinhibition of pyramidal neuron dendrites was proposed theoretically ${ }^{9}$ and has received strong empirical support from a series of recent experiments ${ }^{5-8,14,15,39}$. Here we explored the functional roles of dendritic disinhibition using computational modelling, at both the single-neuron and circuit levels. In contrast to somatic disinhibition, dendritic disinhibition can gate the inputs to a neuron $9,11,31$. We propose that dendritic disinhibition can be utilized to gate inputs from separate pathways, by specifically disinhibiting dendrites that receive inputs from a target pathway.

We studied the effectiveness of gating in an interneuronal circuit model. Most data used to constrain the model have become available only in recent years thanks to the advance of optogenetics and other experimental tools. Where data are not available, we considered the 'worst-case scenario', namely, connections from VIP to SOM neurons, and from SOM to pyramidal dendrites are completely random, which is most likely not the case ${ }^{40}$ and any specificity would facilitate our proposed mechanism. Although the SOM-to-pyramidal connections are dense, we found that the connectivity from SOM neurons to pyramidal dendrites is actually sparse enough to support branchspecific disinhibition. We found that the increase of somatic inhibition mediated by the SOM-PV-pyramidal neuron connections further improves gating selectivity. We demonstrated that branch-specific clustering of excitatory pathways can naturally emerge from disinhibitory regulation of synaptic plasticity. As proof of principle, we applied this mechanism to a model for a recent experiment using a context-dependent decision-making task ${ }^{28}$.

Inhibitory connections in the cortex tend to be dense ${ }^{27}$. This finding has led to the proposal that cortical inhibition functions as a locally non-selective 'blanket of inhibition'29. Our study offers an alternative perspective, which is compatible with dense interneuronal connectivity, but has different implications for circuit functions. The dense connectivity is measured on a cell-tocell level. Nonetheless, connections from dendrite-targeting SOM interneurons can be sparse at the level of the dendritic branch, and therefore potentially selective as required for our gating scheme. Our alternative proposal is fundamentally grounded in consideration of dendritic branches as functional units of computation $^{20}$.

Our hypothesis has three essential requirements. First, dendritic inhibition must be able to effectively regulate dendritic processing of excitatory inputs. This has been shown in our simulation of a fully reconstructed and a simplified neuron model (Supplementary Fig. 1; Fig. 2), as well as in previous models ${ }^{31}$.

The second requirement states that dendritic disinhibition should be specific at the level of dendritic branches. In a simplified interneuronal circuit model, we showed that the SOM-to-pyramidal neuron circuit can very well support branchspecific disinhibition, mainly because the connectivity from SOM neurons to pyramidal dendrites is effectively sparse. Several circuit components are identified as critical to this requirement and subjects to experimental tests, including the number of SOM neurons targeting each dendrite, and the proportion of SOM neurons targeted by top-down control inputs.

The third and final requirement is an alignment between excitation and disinhibition, which we found can be achieved through synaptic plasticity on excitatory synapses. This feature could also potentially be achieved through inhibitory plasticity ${ }^{41}$, by adapting the disinhibition pattern to align with fixed excitatory inputs. These two forms of plasticity are complementary, and both are likely at play. Indeed, a recent study found that during motor learning, spine reorganization on dendrites of pyramidal neurons is accompanied by change in the number of SOM-neuron synapses onto these dendrites ${ }^{40}$. One appeal of studying excitatory plasticity here is that our calcium-based plasticity model could be quantitatively constrained by data, and therefore tested in a biologically plausible regime. At present, much less is known experimentally about the dependence of inhibitory plasticity on pre- and post-synaptic spike timing, dendritic calcium levels or the class of interneuron ${ }^{42}$.

Although all necessary, the three requirements only need to be fulfilled to a certain degree. For example, dendritic inhibition needs to effectively regulate, but need not abolish, the effects of excitatory inputs. It would be of interest to investigate which requirements pathway-specific gating is most sensitive to, in future research.

A direct consequence of the branch-specific disinhibition and excitation-disinhibition alignment is branch-specific excitation, namely, clustering of excitatory pathways onto pyramidal neuron dendritic branches. The computational benefits of input clustering have been previously proposed ${ }^{43}$. There is mounting experimental evidence for input clustering, from anatomical and physiological studies ${ }^{23,24}$ (for a review see ref. 25). Consistent with our model, experimental studies have shown that input clustering can emerge through NMDAR-dependent synaptic plasticity 44 , and that clustering is functionally related to learning 24,45 . Our model prediction that branch-specific clustering can occur at the level of pathways remains to be directly tested.

We focused on whether the disinhibitory circuit motif can support pathway-specific gating. Our model contains only the minimal elements to answer this question. We did not include all known connections between the four major classes of neurons. In particular, among the missing connections are the pyramidal-tointerneuron and the SOM-to-VIP connections. These connections are unlikely to influence our results, since none affect our essential requirements. It remains to be tested whether the pyramidal-to-pyramidal recurrent excitation will interfere with the plasticity-based induction of excitation-disinhibition alignment. We did not model synaptic dynamics or short-term plasticity, because we focused on the steady-state behaviour.

Our model makes specific, experimentally testable predictions. One of the most straightforward and testable predictions is that SOM neurons should show context/rule selectivity in some context-dependent or rule-based tasks. Surprisingly, we found that VIP neurons need not be context-selective, as long as SOM neurons are directly receiving context-selective excitatory control inputs (Fig. 5d-f). Experimental disruption of these contextselective interneurons should impair the animal's ability to perform context- or rule-dependent choice tasks. The contextselectivity of SOM or VIP neurons is not necessarily present in every behavioural task. For instance, a recent study, recording in mouse prefrontal cortex during a auditory discrimination task, found highly homogeneous responses within SOM and VIP populations ${ }^{12}$. We propose that SOM neurons are more likely to exhibit selectivity to context or task in experiments in which the animal performs multiple tasks and branch-specific dendritic spikes also exhibit task selectivity ${ }^{17,46}$. A direct test of our model awaits future experiments in a task-switching paradigm to examine gating of different pathways into association cortical areas and the selective changes of activity in SOM neural subpopulations. We emphasize that interneuron classes in our model should be more appropriately interpreted according to their projection targets rather than their biochemical markers. 
Branch-specific dendritic spikes are already observed experimentally, and SOM neurons are critical for this branchspecificity ${ }^{46}$. It is however unknown whether SOM-mediated inhibition is also branch-specific. Direct patch-clamping of pyramidal neuron dendrites in vivo ${ }^{47}$ can isolate inhibitory currents on individual branches, and provide a direct test for our hypothesis, although such an experiment is technically difficult at present. Our plasticity model predicts that SOM interneurons play a critical role in the learning-related emergence of branchspecific clustering of excitatory synapses on pyramidal neuron dendrites $^{24}$.

Flexible gating, or routing, of information has been a longstanding problem in computational neuroscience ${ }^{48}$, for which a number of models have been proposed. Among proposed ideas are dynamic synaptic weight modulation ${ }^{49}$, gain modulation ${ }^{48}$, synchrony in the input signals ${ }^{18}$, perfect balance of excitation and inhibition $^{19}$,

up/down state-switch in dendrites ${ }^{50}$, switching between different neural pools that receive inputs from distinct pathways ${ }^{51}$ and rule signalling as a selection vector ${ }^{28}$. Notably, most of these models implement a form of soft gating, which modulates the effective strength of incoming pathways instead of performing a binary on-off switch on them.

These prior models did not exploit the computational power of dendrites (except for ref. 50) or the roles of specialized classes of interneurons. Harnessing dendrites rather than populations of intermediate neurons saves the number of neurons needed by manyfold. Only in the limit of one dendrite per pyramidal neuron does our mechanism become conceptually similar to gating mechanisms operating on the neuronal level ${ }^{51}$.

The gating mechanism as studied here is nonlinear but not binary. Typically, the gating selectivity is 0.5 or lower (Figs 4 and 5 ), whereas it would be one if gating was perfect. In the biologically plausible regime of inhibitory strength studied here, shunting inhibition on a dendritic branch still allows synaptic input to appreciably elevate the dendritic voltage and thus impact the soma, which decreases the gating selectivity of the neuron. Gating selectivity is also limited by the number of dendritic branches (or more generally, quasi-independent computational units) on a pyramidal neuron, which is about two dozen. Thus, the proposed mechanism may be especially suitable for 'pathwayspecific gating'. Multiple mechanisms may jointly contribute to gating function, and our proposed mechanism is most likely compatible with the aforementioned proposals.

A testable prediction of our model is that a behavioural context or rule guiding routing of information engages top-down signalling that targets specific classes of VIP and SOM inhibitory neurons. This is in contrast to the conventional thinking of executive control as mediated by top-down control signals to pyramidal cells. The present model suggests that a context signal can correspond to a top-down input from a brain area presenting task context onto VIP and SOM cells (leading to effective excitation or inhibition, respectively, of pyramidal neurons).

To conclude, our findings suggest a microcircuit architecture that harnesses dendritic computation and diverse inhibitory neuron types to subserve cognitive flexibility.

\section{Methods}

All models. A summary of all types of models used and where they are used can be found in Supplementary Table 2.

Spiking pyramidal neuron models. For the fully reconstructed multi-compartmental pyramidal neuron model (Supplementary Fig. 1a-d), we adapted a previously developed model based on a layer $2 / 3$ pyramidal neuron in the rat somatosensory cortex reported by Branco et al. ${ }^{52}$. We used the passive membrane parameter set; results are essentially the same with the active membrane parameter set. Simulations were implemented with the NEURON simulator ${ }^{53}$.
The reduced multi-compartmental spiking neuron model is comprised of multiple dendritic compartments and one somatic compartment. All dendritic compartments are equivalent, not directly coupled to each other, and coupled to the soma. There are 10 dendritic compartments for all simulations using this model (Figs 2 and 7). The number of dendrites does not change the results as long as we normalize the dendrite-soma coupling strength with respect to the number of dendrites. The soma is modelled as a leaky-integrate-and-fire compartment with dynamics following:

$$
C_{\mathrm{S}} \frac{\mathrm{d} V_{\mathrm{S}}}{\mathrm{d} t}=-g_{\mathrm{L}, \mathrm{S}}\left(V_{\mathrm{S}}-E_{\mathrm{L}}\right)-\sum_{i} g_{\mathrm{c}}\left(V_{\mathrm{S}}-V_{i, \mathrm{D}}\right)+I_{\mathrm{syn}, \mathrm{S}}
$$

where the subscripts $\mathrm{S}$ and $\mathrm{D}$ correspond to soma and dendrites, respectively. $V_{i, \mathrm{D}}$ is the membrane potential of the $i$ th dendrite. $C_{S}$ is the membrane capacitance, $E_{\mathrm{L}}$ is the resting potential, $g_{\mathrm{L}}$ is the leak conductance and $g_{\mathrm{c}}$ is the coupling between each dendritic compartment and the somatic compartment. We set $C_{\mathrm{S}}=50.0 \mathrm{pF}$ and $g_{\mathrm{L}, \mathrm{S}}=2.5 \mathrm{nS}$, producing a $20-\mathrm{ms}$ membrane time constant for the soma. We also set $E_{\mathrm{L}}=-70 \mathrm{mV}$ and $g_{\mathrm{c}}=40 \mathrm{nS}$. The somatic spiking mechanism is integrateand-fire, with spike threshold $-50 \mathrm{mV}$, reset potential $-55 \mathrm{mV}$ and refractory period $2 \mathrm{~ms}$. The dynamics of the dendritic membrane potential $\left(V_{\mathrm{D}}\right)$ follows

$$
C_{\mathrm{D}} \frac{\mathrm{d} V_{\mathrm{D}}}{\mathrm{d} t}=-g_{\mathrm{L}, \mathrm{D}}\left(V_{\mathrm{D}}-E_{\mathrm{L}}\right)-g_{\mathrm{c}}\left(V_{\mathrm{D}}-\hat{V}_{\mathrm{S}}\right)+I_{\mathrm{syn}, \mathrm{D}}
$$

where $\hat{V}_{\mathrm{S}}$ is the somatic shadow potential ${ }^{54}$, which follows the same equation as $V_{\mathrm{S}}$, except with no spiking and resetting. We set $C_{\mathrm{D}}=20.0 \mathrm{pF}$ and $g_{\mathrm{L}, \mathrm{D}}=4.0 \mathrm{nS}$, producing a 5 -ms membrane time constant ${ }^{55}$. After a somatic spike, the backpropagating action potential is modelled as a 3 -ms delayed voltage increase of $10 \mathrm{mV}$ in all dendrites ${ }^{56}$.

The main free parameters of the reduced compartmental model, $g_{\mathrm{c}}$ and $g_{\mathrm{L}, \mathrm{D}}$, were chosen to match in vitro properties reported by Nevian $e t a l^{55}$. Specifically, a single-synapse dendritic EPSP of $1-\mathrm{mV}$ peak is attenuated to $\sim 0.05 \mathrm{mV}$ in the soma, and a dendritic NMDA plateau potential evokes a somatic depolarization with the peak $\sim 10 \mathrm{mV}$. We also made several efforts to adapt our model to mimic physiological in vivo conditions, including excitation-inhibition balanced background inputs and reduced soma-dendrite coupling. We used an in vivo set of parameters whenever appropriate (Fig. 2d-g; Fig. 7c,d). The soma-dendrite coupling is reduced fivefold to $g_{c, v i v o}=0.8 \mathrm{nS}$, to achieve the stronger signal attenuation observed in high-conductance state ${ }^{57}$. In this regime, the soma also receives excitatory and inhibitory background inputs, $500 \mathrm{~Hz}$ of $2.5-\mathrm{nS}$ AMPAR input and $150 \mathrm{~Hz}$ of 4.0-nS GABAR input, to approximate the excitationinhibition balanced background input that gives the neuron a baseline Poisson-like firing rate $\sim 3 \mathrm{~Hz}$. Reduced spiking neuron simulations were implemented with the BRIAN neural simulator ${ }^{58}$.

We used four types of synapses, AMPAR, NMDAR, GABA ${ }_{A} R$ and $G A B A_{B} R$ Since $\mathrm{GABA}_{\mathrm{B}} \mathrm{Rs}$ are only used briefly in (Supplementary Fig. 3), we denote $\mathrm{GABA}_{\mathrm{A}}$ simply as GABA. AMPAR and GABAR synapses are modelled as linear:

$$
\begin{gathered}
I_{\mathrm{syn}}=-\tilde{g}_{\mathrm{syn}} s_{\mathrm{syn}}\left(V-E_{\mathrm{syn}}\right) \\
\frac{\mathrm{d} s_{\mathrm{syn}}}{\mathrm{d} t}=-\frac{s_{\mathrm{syn}}}{\tau_{\mathrm{syn}}}+\sum_{i} \delta\left(t-t_{i}\right),
\end{gathered}
$$

where $s_{\text {syn }}$ is the gating variable representing the proportion of open channels, $\tilde{g}_{\text {syn }}$ is the maximum synaptic conductance, $E_{\mathrm{syn}}$ is the synaptic reversal potential, $\tau_{\text {syn }}$ is the synaptic time constant and $t_{i}$ are pre-synaptic spike times. We set $\tau_{\mathrm{AMPA}}=2 \mathrm{~ms}, E_{\mathrm{AMPA}}=E_{E}=0 \mathrm{mV}, E_{\mathrm{GABA}}=E_{\mathrm{I}}=-70 \mathrm{mV}$ and $\tilde{g}_{\mathrm{GABA}}=4.0 \mathrm{nS}$. For dendrite-targeting inhibitory synapses, $\tau_{\mathrm{GABA} \text {,dend }}=20 \mathrm{~ms}$, whereas $\tau_{\mathrm{GABA} \text {,soma }}=10 \mathrm{~ms}$ for soma-targeting inhibitory synapses. These are based on the observations that dendrite-targeting inhibition tend to be slower ${ }^{59,60}$. In Supplementary Fig. 1d,h, $\tilde{g}_{\mathrm{AMPA}}=2.5 \mathrm{nS}$. In Supplementary Fig. 3, $\tilde{g}_{\mathrm{AMPA}}$ ranges from 0 to $2.5 \mathrm{nS}$. Otherwise $\tilde{g}_{\mathrm{AMPA}}$ is set as $0 \mathrm{nS}$ (no AMPAR input).

$\mathrm{GABA}_{\mathrm{B}} \mathrm{R}$ synapses are post-synaptic. Each spike at time $t_{i}$ increases the gating variable $s_{\mathrm{GABA}_{\mathrm{B}}}(t)$ by $\gamma_{\mathrm{GABA}_{\mathrm{B}}}\left[\exp \left[\left(t-t_{i}\right) / \tau_{\mathrm{GABA}_{\mathrm{B}} \text {,decay }}\right]-\exp \left[\left(t-t_{i}\right) / \tau_{\mathrm{GABA}_{\mathrm{B}} \text {,rise }}\right]\right]$, where $\gamma_{\mathrm{GABA}_{\mathrm{B}}}$ is a normalizing factor such that the peak of the above expression is 1. Then, the total input current voltage dependent is

$$
I_{\mathrm{GABA}_{\mathrm{B}}}=-\tilde{g}_{\mathrm{GABA}_{\mathrm{B}}} s_{\mathrm{GABA}_{\mathrm{B}}} f_{\mathrm{GABA}_{\mathrm{B}}}(V)
$$

where $f_{\mathrm{GABA}_{\mathrm{B}}}(V)=33.33 \mathrm{mV} \cdot(0.5-2 /(1+\exp ((V+98.73) / 12.5)))$, as obtained from ref. 61.

NMDAR synapses include a voltage-dependent magnesium block $f_{\mathrm{Mg}}(V)$ and saturating gating variable $s_{\mathrm{NMDA}}$ :

$$
I_{\mathrm{NMDA}}=-\tilde{g}_{\mathrm{NMDA}} s_{\mathrm{NMDA}}\left(V-E_{E}\right) f_{\mathrm{Mg}}(V)
$$

$$
f_{\mathrm{Mg}}(V)=\left[1+\exp \left(-\frac{V-V_{\text {half }}}{V_{\text {width }}}\right)\right]^{-1}
$$

with $V_{\text {half }}=-19.9 \mathrm{mV}$ and $V_{\text {width }}=12.48 \mathrm{mV}$ (ref. 62). The NMDA conductance 
$\tilde{g}_{\mathrm{NMDA}}=2.5 \mathrm{nS}$. The NMDAR gating variable dynamics follow:

$$
\begin{gathered}
\frac{\mathrm{d} s_{\mathrm{NMDA}}}{\mathrm{d} t}=-\frac{s_{\mathrm{NMDA}}}{\tau_{\mathrm{NMDA}, \text { decay }}}+\alpha_{\mathrm{NMDA}} x_{\mathrm{NMDA}}(t)\left(1-s_{\mathrm{NMDA}}\right) \\
\frac{\mathrm{d} x_{\mathrm{NMDA}}}{\mathrm{d} t}=-\frac{x_{\mathrm{NMDA}}}{\tau_{\mathrm{NMDA}, \text { rise }}}+\sum_{i} \delta\left(t-t_{i}\right)
\end{gathered}
$$

with $\tau_{\mathrm{NMDA}, \text { decay }}=100 \mathrm{~ms}, \tau_{\mathrm{NMDA}, \text { rise }}=2 \mathrm{~ms}$ and $\alpha_{\mathrm{NMDA}}=0.3 \mathrm{~ms}^{-1}$. This choice of $\alpha_{\text {NMDA }}$ sets $s_{\text {NMDA }}$ to be $\sim 0.4$ at its peak after a single spike ${ }^{63,64}$. With this value of $\alpha_{\mathrm{NMDA}}$, the saturation of NMDA starts to get prominent around firing rate $r=1 /\left(\alpha_{\mathrm{NMDA}} \tau_{\mathrm{NMDA}, \text { rise }} \tau_{\mathrm{NMDA}, \text { decay }}\right) \approx 16 \mathrm{~Hz}$. By default in simulations with the reduced spiking model, the excitatory inputs are 15 independent NMDAR synapses with the same rate. Fewer number of excitatory synapses can become insufficient to elicit NMDA plateau potential. Since GABAR and AMPAR synapses are linear, their inputs are directly represented by the overall rates.

Each excitatory synapse also has a calcium concentration level with arbitrary unit, which consists of two components, one NMDAR dependent and one voltagegated calcium channel (VGCC) dependent: $\left[\mathrm{Ca}^{2+}\right]=\left[\mathrm{Ca}^{2+}\right]_{\mathrm{NMDA}}+\left[\mathrm{Ca}^{2+}\right]_{\mathrm{VGCC}}$. The NMDAR-dependent component is modelled as leaky integration of the NMDAR current:

$$
\tau_{\mathrm{Ca}, \mathrm{decay}} \frac{\mathrm{d}\left[\mathrm{Ca}^{2+}\right]_{\mathrm{NMDA}}}{\mathrm{d} t}=-\left[\mathrm{Ca}^{2+}\right]_{\mathrm{NMDA}}+\kappa_{\mathrm{NMDA}} I_{\mathrm{NMDA}},
$$

where $\kappa_{\mathrm{NMDA}}$ is a scaling parameter with unit per pA. The VGCC component is evoked by post-synaptic spikes that back-propagate into dendrites. Each spike induces a bi-exponential increase:

$$
\left[\mathrm{Ca}^{2+}\right]_{\mathrm{VGCC}}(t)=\kappa_{\mathrm{VGCC}} \gamma_{\mathrm{Ca}} \sum_{i}\left[\exp \left(-\frac{t-t_{i}}{\tau_{\text {Ca,decay }}}\right)-\exp \left(-\frac{t-t_{i}}{\tau_{\mathrm{Ca}, \text { rise }}}\right)\right] .
$$

Here $\gamma_{\mathrm{Ca}}$ is a normalizing constant so that the peak response to one spike is $\kappa_{\mathrm{VGCC}}$ And $\kappa_{\mathrm{VGCC}}$ is again a scaling parameter. $\tau_{\mathrm{Ca}}$,decay $=30 \mathrm{~ms}$ is estimated from ref. 37 . $\tau_{\text {Ca,rise }}=2 \mathrm{~ms}$ is used mainly to make $\left[\mathrm{Ca}^{2}+\right]$ continuous.

NMDA plateau potential. The voltage of a dendrite receiving NMDAR and GABAR inputs follows

$$
\begin{aligned}
C_{\mathrm{D}} \frac{\mathrm{d} V_{\mathrm{D}}}{\mathrm{d} t} & =-g_{\mathrm{L}, \mathrm{D}}\left(V_{\mathrm{D}}-E_{\mathrm{L}}\right)-g_{\mathrm{c}}\left(V_{\mathrm{D}}-\hat{V}_{\mathrm{S}}\right) \\
& -\sum_{j} \tilde{g}_{\mathrm{NMDA}} s_{\mathrm{NMDA}, j}(t)\left(V_{\mathrm{D}}-E_{\mathrm{E}}\right) f_{\mathrm{Mg}}\left(V_{\mathrm{D}}\right)-\sum_{k} \tilde{g}_{\mathrm{GABA}} s_{\mathrm{GABA}, k}(t)\left(V_{\mathrm{D}}-E_{\mathrm{I}}\right),
\end{aligned}
$$

where $j$ and $k$ are indices of NMDAR and GABAR synapses, respectively. Denote

$$
g_{\mathrm{NMDA}}(t)=\sum_{j} \tilde{g}_{\mathrm{NMDA}} s_{\mathrm{NMDA}, j}(t)
$$

as the total NMDA input conductance onto this dendrite. The maximum value of $g_{\mathrm{NMDA}}(t)$ is simply $g_{\mathrm{NMDA}, \max }=\sum_{j} \tilde{g}_{\mathrm{NMDA}}=N_{\mathrm{NMDA}} \tilde{g}_{\mathrm{NMDA}}$, where $N_{\mathrm{NMDA}}$ is the number of NMDAR synapses. Similarly,

$$
g_{\mathrm{GABA}}(t)=\sum_{k} \tilde{g}_{\mathrm{GABA}} s_{\mathrm{GABA}, k}(t) .
$$

If we ignore the coupling between this dendrite and its soma for now, and consider constant synaptic conductances $g_{\mathrm{NMDA}}=g_{\mathrm{NMDA}}(t), g_{\mathrm{GABA}}=g_{\mathrm{GABA}}(t)$. Then, we have

$$
C_{\mathrm{D}} \frac{\mathrm{d} V_{\mathrm{D}}}{\mathrm{d} t}=-g_{\mathrm{L}, \mathrm{D}}\left(V_{\mathrm{D}}-E_{\mathrm{L}}\right)-g_{\mathrm{NMDA}}\left(V_{\mathrm{D}}-E_{\mathrm{E}}\right) f_{\mathrm{Mg}}\left(V_{\mathrm{D}}\right)-g_{\mathrm{GABA}}\left(V_{\mathrm{D}}-E_{\mathrm{I}}\right) .
$$

Since we have $E_{\mathrm{I}}=E_{\mathrm{L}}$, the steady-state dendritic voltage $V_{\mathrm{D} \text {,ss }}$ satisfies

$$
0=-\left(V_{\mathrm{D}, \mathrm{ss}}-E_{\mathrm{L}}\right)-\frac{g_{\mathrm{NMDA}}}{g_{\mathrm{L}, \mathrm{D}}+g_{\mathrm{GABA}}}\left(V_{\mathrm{D}, \mathrm{ss}}-E_{\mathrm{E}}\right) f_{\mathrm{Mg}}\left(V_{\mathrm{D}}\right) .
$$

This equation can be solved numerically, resulting in the curve in Supplementary Fig. 2d.

Pathway-specific gating in a single pyramidal neuron. Gating is performed by disinhibiting a specific subset of dendrites. Disinhibited dendrites always receive $5 \mathrm{~Hz}$ background inhibition. The disinhibition level is defined as the difference between the rates of inhibition received by inhibited and disinhibited dendrites.

In Fig. 2d-g, each pathway targets two dendrites with 15 NMDAR synapses on each dendrite. The dendrites targeted by each pathway do not overlap. For each pathway, the input rate $\left(u_{\mathrm{E}}\right)$ follows a bell-shaped tuning to the stimulus value $(z)$ : $u_{\mathrm{E}}=40 \exp \left(-z^{2}\right) \mathrm{Hz}$, where $z$ ranges between -2.4 and 2.4. The disinhibition level is $30 \mathrm{~Hz}$ (from 35 to $5 \mathrm{~Hz}$ ).

Presented alone, the preferred stimulus $(z=0)$ from one pathway increases the output firing rate by $r_{\text {on }}\left(r_{\text {off }}\right)$ when the pathway is gated-on (off). The gating selectivity is defined as

$$
\frac{r_{\text {on }}-r_{\text {off }}}{r_{\text {on }}+r_{\text {off }}},
$$

For Fig. 3, excitatory pathways can overlap. In the context with gate 1 open, $N_{\text {disinh }}$ dendrites are disinhibited. Excitatory pathway 1 targets these $N_{\text {disinh }}$ dendrites, each with strength $25 \mathrm{nS}$, and similarly for gate 2 and pathway 2 . The $N_{\text {disinh }}$ dendrites disinhibited for gate 2 are chosen randomly and independently from the $N_{\text {disinh }}$ dendrites disinhibited for gate 1 . For each $N_{\text {disinh }}$ and $N_{\text {dend }}, r_{\text {on }}$ and $r_{\text {off }}$ are averaged across all possible projection patterns.

Rate pyramidal neuron model. The rate model is fitted with simulation data from the spiking model with in vivo parameters (Supplementary Fig. 4). The timeaveraged voltage of a dendritic compartment $\left(\bar{V}_{\mathrm{D}}\right)$ is modelled as a sigmoidal function of total excitatory input conductance $\left(\bar{g}_{\mathrm{E}}\right.$, see below for definition) following:

$$
\bar{V}_{\mathrm{D}}=f_{\mathrm{V}}\left(\bar{g}_{\mathrm{E}}, \bar{g}_{\mathrm{I}}\right)=30 \cdot\left[1+\tanh \left(\frac{\bar{g}_{\mathrm{E}}-g_{1 / 2}}{\beta}\right)\right]+V_{0}+E_{\mathrm{L}} .
$$

The mid-point $g_{1 / 2}$ is proportional to the total inhibitory conductance $\bar{g}_{\text {I }}$ plus the leak conductance of the dendrite $g_{\mathrm{L}, \mathrm{D}}$, as expected from the constant conductance scenario (Supplementary Fig. 2c)

$$
g_{1 / 2}=b_{\mathrm{g}} \cdot\left(g_{\mathrm{L}, \mathrm{D}}+\bar{g}_{\mathrm{I}}\right) \text {. }
$$

On the basis of our observation of the reduced spiking model, we modelled the width $\beta$ as an exponentially increasing function of inhibition:

$$
\beta=k \cdot \exp \left(\bar{g}_{\mathrm{I}} / \gamma\right) \text {. }
$$

This increase of width $\beta$ as a function of $\bar{g}_{\mathrm{I}}$ captures the linearization effect of sparse inhibition on the voltage input function (Fig. 2c). Fit values of the parameters are $b_{\mathrm{g}}=5.56, k=9.64 \mathrm{nS}, \gamma=6.54 \mathrm{nS}$ and $V_{0}=0.78 \mathrm{mV}$. The model is fitted to a simulated 10-dendrite spiking neuron model. When simulating dendrites of the spiking model, somatic shadow voltage is clamped at $-60 \mathrm{mV}$, and back-propagating action potential is fixed as a Poisson spike train of $10 \mathrm{~Hz}$. This phenomenological model allows us to interpolate the dendritic voltage for a large range of excitatory and inhibitory inputs very rapidly.

The firing rate of the soma is modelled as a power law function of input current

$$
r=f_{r}(I)=[\max (0, I+174.86) / 45.16]^{2.89}
$$

Here $I$ is the sum of the input current from dendrites and also the somatic inhibition from PV neurons whenever applicable. The parameters are fitted from simulation of the reduced spiking model. We assume the somatic voltage fluctuates around $E_{\text {reset }}$, and denote the mean dendritic voltage $\left\langle V_{\mathrm{D}}\right\rangle$. Then, the input current from dendrites is $I_{\text {dend } \rightarrow \text { soma }}=G_{\mathrm{c}} \cdot\left(\left\langle V_{\mathrm{D}}\right\rangle-E_{\text {reset }}\right)$, where $G_{\mathrm{c}}$ is the total dendritesoma coupling of all dendrites. $G_{\mathrm{c}}=8 \mathrm{nS}$. Since we assume $G_{\mathrm{c}}$ is fixed whenever we vary the number of dendrites (Figs 3 and 4), the somatic function does not depend on the number of dendrites and need not be re-parametrized. So $I=I_{\text {dend } \rightarrow \text { soma }}$ $+\Delta I_{\mathrm{PV} \rightarrow \text { soma }}$, where $\Delta I_{\mathrm{PV} \rightarrow \text { soma }}$ is the change in somatic inhibition from PV neurons.

For inputs to the rate model, $\bar{g}_{\mathrm{E}}$ and $\bar{g}_{\mathrm{I}}$ are the time-averaged total conductance of all excitatory and inhibitory synapses, respectively. For NMDAR-only excitatory input, the approximated time-averaged gating variable $\bar{s}_{\mathrm{NMDA}}$ of a single synapse receiving input rate $r_{\mathrm{E}}$ follows,

$$
\bar{s}_{\mathrm{NMDA}}=1-\left(1+r_{\mathrm{E}} \tau_{\mathrm{NMDA}, \text { rise }} \tau_{\mathrm{NMDA}, \text { decay }} \alpha_{\mathrm{NMDA}}\right)^{-1} .
$$

For $N_{\mathrm{NMDA}}$ synapses each with maximal conductance $\tilde{g}_{\mathrm{NMDA}}$, the total excitatory conductance is

$$
\bar{g}_{\mathrm{E}}=N_{\mathrm{NMDA}} \bar{s}_{\mathrm{NMDA}} \tilde{g}_{\mathrm{NMDA}} .
$$

Therefore, $\bar{g}_{\mathrm{E}}$ saturates as $\bar{s}_{\mathrm{NMDA}}$ does. Because the GABAR conductance is linear in its input rate, the total inhibitory conductance is

$$
\bar{g}_{\mathrm{I}}=r_{\mathrm{I}} \tau_{\mathrm{GABA}} \tilde{g}_{\mathrm{GABA}}
$$

where $r_{\mathrm{I}}$ is the overall inhibitory input rate onto that dendrite.

Interneuron models. SOM neurons are modelled as simple rate neurons with a rectified linear $\mathrm{f}-\mathrm{I}$ curve. The firing rate of a SOM neuron is

$$
r_{\mathrm{SOM}}=\max \left(0, \beta_{\mathrm{SOM}}\left(I_{\mathrm{syn}, \mathrm{SOM}}-I_{\mathrm{rh}, \mathrm{SOM}}\right)\right),
$$

where $\max (x, 0)$ is a rectified linear function of $x . I_{\mathrm{rh}, \mathrm{SOM}}=40 \mathrm{pA}$ is the rheobase, that is, the minimum current required to activate the neuron, and $\beta_{\mathrm{SOM}}=90 \mathrm{~Hz} /$ $\mathrm{nA}$ is the $\mathrm{f}-\mathrm{I}$ curve slope for SOM neurons, which we matched to data from ref 65 . SOM neurons typically display adapting responses to constant input and the synapses of SOM neurons show short-term plasticity. We ignored these aspects of temporal dynamics because here we are interested in the steady-state response. SOM neurons receive $150 \mathrm{pA}$ input current in the default state, leading to a baseline firing of SOM neurons $\sim 10 \mathrm{~Hz}$ as observed experimentally ${ }^{6,15}$.

For VIP neurons, we assume that the control input targets $N_{\text {control,VIP }}=\operatorname{round}\left(N_{\mathrm{VIP}} \cdot P_{\text {control,VIP }}\right)$ of them. On average, VIP neurons are assumed to fire at $\bar{r}_{\mathrm{VIP}}=5 \mathrm{~Hz}$. Therefore, the VIP neurons non-activated by the 
control input fire at $0 \mathrm{~Hz}$, while those targeted by the control input fire at $\left(5 \cdot N_{\text {VIP }} / N_{\text {control,VIP }}\right) \mathrm{Hz}$.

PV neurons are modelled simply as linear rate neurons with a f-I curve slope of $\beta_{\mathrm{PV}}=220 \mathrm{~Hz} \mathrm{nA}^{-1}$, because their activities never reach zero in our model. Since we are only interested in their change of activities in response to SOM neuron suppression, the spontaneous activity of PV neurons is irrelevant to our model.

Interneuronal network. The full interneuronal network model contains pyramidal, SOM, VIP and PV neurons. The network model is roughly based on a L2/3 cortical column microcircuit, and contains 3,000 pyramidal neurons, $160 \mathrm{SOM}$ neurons, 140 VIP neurons and $200 \mathrm{PV}$ neurons ${ }^{66}$. However, the analysis applies more generally. Pyramidal neurons are modelled as multi-compartmental rate neurons as described above. We typically used $N_{\text {dend }}=30$ dendrites, approximately corresponding to pyramidal neurons in associative areas ${ }^{67}$. The number of pyramidal neurons does not affect our results.

The SOM-to-dendrite connections are set randomly. Instead of drawing each connection randomly and independently with a fixed probability, we assume that each dendrite is targeted by precisely $N_{\mathrm{SOM} \rightarrow \text { dend }} \mathrm{SOM}$ neurons, when $N_{\mathrm{SOM} \rightarrow \text { dend }}$ is an integer, so that each dendrite receives the same amount of net inhibition in the default state. The identities of SOM neurons targeting each dendrite are randomly chosen. The total inhibitory conductance received by each dendrite is denoted and fixed as $G_{\mathrm{SOM} \rightarrow \text { dend }}=40 \mathrm{nS}$, then for each SOM-dendrite connection the conductance is $G_{\mathrm{SOM} \rightarrow \text { dend }} / N_{\mathrm{SOM} \rightarrow \text { dend. }}$. Each SOM-dendrite connection can contain multiple synapses, which we are not explicitly modelling here because GABAergic synapses are linear such that we only need to consider the total conductance. When $N_{\mathrm{SOM} \rightarrow \text { dend }}$ is not an integer, we interpolate. Each dendrite is targeted by $\left\lceil N_{\mathrm{SOM} \rightarrow \text { dend }}\right\rceil$ SOM neurons, where all synapses but one have weights $G_{\mathrm{SOM} \rightarrow \text { dend }} / N_{\mathrm{SOM} \rightarrow \text { dend, }}$ while one has weight $G_{\mathrm{SOM} \rightarrow \text { dend }} \cdot\left(1-\left\lfloor N_{\mathrm{SOM} \rightarrow \text { dend }}\right\rfloor /\right.$ $\left.N_{\mathrm{SOM} \rightarrow \text { dend }}\right)$. Given the connection probability from SOM to pyramidal neurons $P_{\mathrm{SOM} \rightarrow \mathrm{pyr}}$, the number of SOM neurons $N_{\mathrm{SOM}}$, and the number of dendrites on each pyramidal neuron $N_{\text {dend }}$, we set

$$
N_{\mathrm{SOM} \rightarrow \text { dend }}=N_{\mathrm{SOM}} \cdot\left[1-\left(1-P_{\mathrm{SOM} \rightarrow \text { pyr }}\right)^{N_{\text {dend }}}\right] .
$$

This is the mean number of SOM neurons targeting each dendrite if the SOM-to-pyramidal connections were completely independent and random.

The VIP-to-SOM connections are set in the same way as the SOM-to-dendrite connections. Since SOM neurons only have one compartment each, we have $N_{\mathrm{VIP} \rightarrow \mathrm{SOM}}=N_{\mathrm{VIP}} \cdot P_{\mathrm{VIP} \rightarrow \mathrm{SOM}}$. When control inputs target both VIP and SOM neurons, we have $P_{\mathrm{VIP}} \rightarrow \mathrm{SOM}=0.6$. When control inputs only target VIP neurons, we have $P_{\mathrm{VIP} \rightarrow \mathrm{SOM}}=0.1$. Within $100 \mu \mathrm{m}$, the connection probability is measured to be $\sim 0.6$ (Supplementary Table 1 ). However, note that the connection probability from VIP to SOM neurons on a column scale is unknown. The spatially restricted axonal arbours of VIP neurons ${ }^{33}$ suggest that the connection probability may fall quickly as a function of the VIP-SOM distance. Therefore, on the scale of a column, the connection probability could still be as low as 0.1 . Total inhibitory connection weight from VIP neurons received by each SOM neuron is

$30 \mathrm{pA} \mathrm{Hz}^{-1}$, and is distributed onto each synapse the same way SOM-to-dendrite connection weights are set. For $N_{\mathrm{VIP}}=140$ and $P_{\mathrm{VIP} \rightarrow \mathrm{SOM}}=0.6$, the connection strength of each synapse is $\sim 0.4 \mathrm{pA} / \mathrm{Hz}$. This is close to the unitary VIP-to-SOM inhibitory post-synaptic charge (IPSQ) of $0.7 \mathrm{pA} \mathrm{Hz}^{-1}$ measured in ref. 5. Notice here the connection is current-based because SOM neurons are described by a f-I curve.

The SOM-to-PV, PV-to-PV and PV-to-pyramidal soma connections are all set similar to the connections above. We set $P_{\mathrm{SOM} \rightarrow \mathrm{PV}}=0.8, P_{\mathrm{PV} \rightarrow \mathrm{PV}}=0.9$ and $P_{\mathrm{PV} \rightarrow \text { soma }}=0.6$ (ref. 5). The total inhibitory connection strength from SOM neurons to each PV neuron is varied in Fig. $6 \mathrm{~b}$. The total inhibitory connection from $\mathrm{PV}$ neurons to each $\mathrm{PV}$ neuron is $30 \mathrm{pA} \mathrm{Hz}^{-1}$, and from $\mathrm{PV}$ neurons to each pyramidal neuron is $30 \mathrm{pA} \mathrm{Hz}^{-1}$. Denote the resulting connection weight matrix $W_{\mathrm{SOM} \rightarrow \mathrm{PV}}, W_{\mathrm{PV} \rightarrow \mathrm{PV}}$ and $W_{\mathrm{PV} \rightarrow \text { soma }}$ then in steady state the change in somatic inhibition $\Delta \mathbf{I}_{\mathrm{pyr}}$ across pyramidal neurons is

$$
\Delta \mathbf{I}_{\text {soma }}=W_{\mathrm{PV} \rightarrow \text { soma }} \Delta \mathbf{r}_{\mathrm{PV}},
$$

where $\Delta \mathbf{r}_{\mathrm{PV}}$ is the change in PV activities. And we have

$$
\Delta \mathbf{r}_{\mathrm{PV}}=\left(\mathbf{1} / \beta_{\mathrm{PV}}-W_{\mathrm{PV} \rightarrow \mathrm{PV}}\right)^{-1} W_{\mathrm{SOM} \rightarrow \mathrm{PV}} \Delta \mathbf{r}_{\mathrm{SOM}},
$$

where $\Delta \mathbf{r}_{\mathrm{SOM}}$ is the change in SOM activities before and after control inputs. $\mathbf{1}$ is identity matrix. The precise values of these PV-related parameters do not matter.

Control inputs are excitatory. Here we are agnostic about their origin. They could be locally generated or from long-range projections. Control inputs can target subsets of SOM and VIP neurons. The mean strength of the control inputs across the whole population is always kept as a constant. When control inputs target SOM neurons, $N_{\text {control,SOM }}=\operatorname{round}\left(N_{\mathrm{SOM}} \cdot P_{\text {control,SOM }}\right)$ of SOM neurons are targeted, with current $75 \cdot N_{\mathrm{SOM}} / N_{\text {control, SOM }} \mathrm{pA}$. Therefore, across the whole population, the averaged current input is $75 \mathrm{pA}$. When control inputs target VIP neurons, each of the $N_{\text {control,VIP }}$ targeted VIP neurons fire at $\left(5 \cdot N_{\text {VIP }} / N_{\text {control,VIP }}\right)$ Hz. For Fig. 5a-c when control inputs only target VIP neurons, we set

$P_{\text {control, }, \mathrm{SOM}}=0$ and $P_{\text {control,VIP }}=0.1 . P_{\text {control, }, \text { IP }}$ has to be low otherwise the gating selectivity would be very close to 0 . For Fig. $5 \mathrm{~d}-\mathrm{f}$, when control inputs target both SOM and VIP neurons, $P_{\text {control,SOM }}=0.5$ and $P_{\text {control.VIP }}=0.5$. Setting

$P_{\text {control,SOM }}=0.5$ does not result in the highest gating selectivity. We did not make particular efforts to fine-tune these parameters.
Finally, excitatory inputs carrying stimulus information for one pathway target the corresponding disinhibited dendrites. When we opened the gate for pathway 1 , suppose one dendrite receives averaged inhibitory conductance $\bar{g}_{\mathrm{I}}$. Then, the total excitatory conductance $\bar{g}_{\mathrm{E}}$ from pathway 1 onto this dendrite is

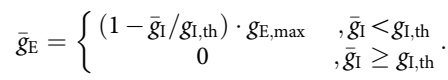

$g_{\mathrm{I}, \text { th }}$ is a inhibitory conductance threshold we defined. Therefore, when inhibition is weak (disinhibition is strong), excitation is inversely proportional to inhibition level. However, when disinhibition is weak, there will be no excitatory input at all. Having a cutoff threshold $g_{\text {I,th }}$ prevents excitatory inputs from targeting every dendrite and therefore being overly dense. We set $g_{\mathrm{I}, \mathrm{th}}=4.0 \mathrm{nS}$. Since we have set the sum of conductances of all inhibitory synapses to be $G_{\mathrm{SOM} \rightarrow \text { dend }}=40 \mathrm{nS}$, each SOM neuron fires $\sim 10 \mathrm{~Hz}$ before disinhibition, and $\tau_{\mathrm{GABA} \text {,dend }}=20 \mathrm{~ms}$, the time-averaged conductance received by each dendrite in default is $\bar{g}_{\mathrm{I}}=r_{\mathrm{I}} \tau_{\mathrm{GABA}, \text { dend }} G_{\mathrm{SOM} \rightarrow \text { dend }}=8.0 \mathrm{nS}$. Therefore, by setting $g_{\mathrm{I}, \mathrm{th}}=4.0 \mathrm{nS}$, excitatory synapses only target dendrites that are at least disinhibited by half. We set the maximum time-averaged excitatory conductance targeting each dendrite to be $g_{\mathrm{E}, \max }=25 \mathrm{nS}$. This value is chosen so that excitation is strong enough to excite a disinhibited dendrite, but not strong enough to excite a strongly inhibited dendrite (Supplementary Fig. 4).

In Supplementary Table 1, we summarized the raw experimental data used to constrain the model.

Synaptic plasticity model and learning protocol. The synaptic plasticity model is calcium-based. The calcium dynamics is described above, and the synaptic weight change given these calcium dynamics is modelled with the formalism from ref. 36, restated below for clarity with slightly modified notations.

Over the time of stimulation, the calcium trace spends time $\alpha_{\mathrm{p}}$ above the potentiation threshold $\theta_{\mathrm{p}}$, and time $\alpha_{\mathrm{d}}$ above the depression threshold $\theta_{\mathrm{d}}$. Then, the average potentiation is $\Gamma_{\mathrm{p}}=\gamma_{\mathrm{p}} \alpha_{\mathrm{p}}$, and the average depression is $\Gamma_{\mathrm{d}}=\gamma_{\mathrm{d}} \alpha_{\mathrm{d}}$, where $\gamma_{\mathrm{p}}$ and $\gamma_{\mathrm{d}}$ are the potentiation and depression strengths, respectively. Since the synapse is assumed to be bistable (DOWN or UP states), denote $\rho$ as the probability of the synapse staying in the UP state, which evolves over time in response to the calcium trace crossing thresholds. Then, define $\bar{\rho}$ as the long-term time average of $\rho$, and $\sigma_{\rho}^{2}$ as the s.d. of $\rho$. Then,

$$
\begin{gathered}
\bar{\rho}=\frac{\Gamma_{\mathrm{p}}}{\Gamma_{\mathrm{p}}+\Gamma_{\mathrm{d}}} \\
\sigma_{\rho}^{2}=\frac{\sigma^{2}\left(\alpha_{\mathrm{p}}+\alpha_{\mathrm{d}}\right)}{\Gamma_{\mathrm{p}}+\Gamma_{\mathrm{d}}},
\end{gathered}
$$

where $\sigma$ is the amplitude of noise and $\tau$ is the time constant of weight change. In long term, the probability to switch from DOWN to UP state $U$ and from UP to DOWN states $D$ are given by

$$
\begin{gathered}
U=\frac{1}{2}\left(1+\operatorname{erf}\left(-\frac{0.5-\bar{\rho}+\bar{\rho} e^{-\left(\Gamma_{\mathrm{p}}+\Gamma_{\mathrm{d}}\right) / \tau}}{\sqrt{\sigma_{\rho}^{2}\left(1-e^{-2\left(\Gamma_{\mathrm{p}}+\Gamma_{\mathrm{d}}\right) / \tau}\right)}}\right)\right) \\
D=\frac{1}{2}\left(1-\operatorname{erf}\left(-\frac{0.5-\bar{\rho}+(\bar{\rho}-1) e^{-\left(\Gamma_{\mathrm{p}}+\Gamma_{\mathrm{d}}\right) / \tau}}{\sqrt{\sigma_{\rho}^{2}\left(1-e^{-2\left(\Gamma_{\mathrm{p}}+\Gamma_{\mathrm{d}}\right) / \tau}\right)}}\right)\right)
\end{gathered}
$$

$\operatorname{erf}(\cdot)$ is the s.e. function. For convenience, we set the weight of DOWN state to $w_{0}=0$ and the weight of UP state $w_{1}=3$. Then, following stimulation, the weight after learning $w_{\text {post }}=w_{\text {pre }}(1-D)+\left(w_{1}-w_{\text {pre }}\right) U$, given the weight before learning $w_{\text {pre. }}$.

We fitted the free parameters of the model with experimental data from ref. 37. In simulation of the plasticity experiment, we modelled the pre-synaptic extracellular stimulation by 40 NMDAR synapses simultaneously receiving one spike. This stimulation alone causes a $2.8 \mathrm{mV}$ depolarization on the soma, which is within the range of observed values $(1-3 \mathrm{mV})$ for that experiment. It also brings the dendrite close to the NMDA plateau threshold, allowing for strong interaction between pre- and post-synaptic spikes. As in the experiment, all pairings are repeated 60 times. The somatic shadow voltage is clamped at $-60 \mathrm{mV}$.

The model is fitted to data points from Figs 2 and $3 \mathrm{~d}$ in ref. 37, and is used to predict the data from Fig. $3 \mathrm{~b}$ therein. Notice that two data points in the test data set (their Fig. 3b) are already included in their Figs 2 and 3d. In all of these cases, there is one pre-synaptic spike, and usually a burst of post-synaptic spikes. The time lag in Supplementary Fig. $7 \mathrm{a}$ is defined as the timing difference between the first postsynaptic spike in the burst and the pre-synaptic spike. In Supplementary Fig. 7a,b, there are three post-synaptic spikes. In Supplementary Fig. 7b,c, the pre-synaptic spike is either $10 \mathrm{~ms}$ earlier than the first post-synaptic spike in the burst or $10 \mathrm{~ms}$ later than the last post-synaptic spike. In Supplementary Fig. 7a,c, the post-synaptic burst, when there is one, has frequency of $50 \mathrm{~Hz}$ (inter-spike interval of $20 \mathrm{~ms}$ ). The fit parameters are the following. The scaling parameters for calcium traces, $\kappa_{\mathrm{NMDA}}=0.371$ and $\kappa_{\mathrm{VGCC}}=0.957$. The depression and potentiation rates are $\gamma_{\mathrm{d}}=39.9$ and $\gamma_{\mathrm{p}}=177.6$. The potentiation threshold for calcium is $\theta_{\mathrm{p}}=2.78$. In fitting this particular data set, we found that there is a certain level of redundancy in parameters; the number of parameters needed to be free is less than the total 
number of potentially free parameters. We therefore fixed two parameters using values in ref 36, which were fitted to another data set: the amplitude of the noise $\sigma=3.35$ and the time constant of synaptic weight change $\tau=346.36 \mathrm{~s}$. The depression threshold is $\theta_{\mathrm{d}}=1$. Before the plasticity-inducing experiment, we have $w_{\text {pre }}=1$ that corresponds to $\tilde{g}_{\mathrm{NMDA}}=2.5 \mathrm{nS}$ for each NMDAR synapse. So after learning, the actual synaptic conductances are $\tilde{g}_{\mathrm{NMDA}}=w_{\text {post }} \cdot 2.5 \mathrm{nS}$.

Just like the spiking pyramidal neuron model, the plasticity model fitted with in vitro data needs to be recalibrated to behave properly under in vivo-like conditions $s^{68}$. We reduced the scaling parameters for calcium traces to $\hat{\kappa}_{\mathrm{NMDA}}=0.75 \kappa_{\mathrm{NMDA}}$, mimicking a reduced extracellular calcium concentration, and to $\hat{\kappa}_{\mathrm{VGCC}}=0.2 \kappa_{\mathrm{VGCC}}$, resembling attenuated effect of back-propagating action potentials in high-conductance in vivo state. These changes also ensure the weights of non-activated synapses do not change substantially throughout the simulation. In Fig. $7 \mathrm{~b}$, the plasticity-inducing protocol is 300 -s long. The post-synaptic firing is fixed at $10 \mathrm{~Hz}$.

In Fig. $7 \mathrm{c}-\mathrm{e}$, inputs from both pathways initially target every dendrite with 15 synapses of the same weight $\tilde{g}_{\mathrm{NMDA}}=2.5 \mathrm{nS}$. Each gate is opened by disinhibiting two distinct dendritic branches. During learning, all synapses from the gated-on pathway are activated at $50 \mathrm{~Hz}$, whereas the gated-off pathway is not activated. The post-synaptic rate is set at $10 \mathrm{~Hz}$. To measure gating selectivity before learning, 8 of the 15 synapses on each dendrite are activated for both pathways. After learning 5 of 15 synapses were activated, the number is chosen so that before and after learning the total excitatory conductance is the same.

Context-dependent decision-making network. We modelled the contextdependent decision-making task from ref. 28. In the experimental task, the stimulus is a mixture of random dots that are leftward- or rightward-moving, and are red or green. The stimulus can be described by its motion and colour coherence. Motion coherence for rightward motion can take six values $(-0.5$, $-0.15,-0.05,0.05,0.15$ and 0.5$)$. Colour coherence for colour red also takes six values $(-0.5,-0.18,-0.06,0.06,0.18$ and 0.5$)$. On each trial, the colour and motion coherence are independently and randomly chosen, resulting in 36 possible stimuli. In Fig. 8d, the performance with respect to motion coherence is averaged across all colour coherences. Similarly for Fig. 8e, the performance with respect to colour coherence is averaged across all motion coherences. In Fig. 8f, the curve for strong motion coherence is averaged across motion coherence -0.5 and 0.5 . Similarly for medium and weak coherences.

The context-dependent decision-making circuit model contains two components. The first is a mixed-selective sensory network, which uses the VIP-SOM-pyramidal neuron circuit model described above. The mixed-selective sensory neurons receive motion and colour inputs from the sensory stimulus. Here the motion and colour inputs do not signal the actual motion and colour of the stimulus, but rather the motion and colour evidence for a particular target. For convenience, the motion direction corresponding to target 1 is denoted left, and the colour corresponding to target 1 is denoted red, and similar for motion right and colour green. This treatment follows the analyses and modelling of ref. 28 . There are four pools of neurons in this network. Each pool prefers a particular combination of motion and colour, for example, left and red. Each neuron pool is modelled exactly as those in Fig. 5d, where the circuit connectivity is random and control inputs target both VIP and SOM neurons, using the base parameter set described above. The input to each dendrites is 15 NMDAR synapses with rate determined by the coherence (coh) of their preferred motion and colour input, as $40 \cdot(1+\mathrm{coh}) \mathrm{Hz}$ (refs 38,69$)$. For example, a neuron that prefers left and red inputs would receive $40 \cdot\left(1+\operatorname{coh}_{\text {left }}\right) \mathrm{Hz}$ input on its dendrites targeted by motion pathway, and $40 \cdot\left(1+\mathrm{coh}_{\mathrm{red}}\right) \mathrm{Hz}$ on its dendrites targeted by colour pathway. Note that $\mathrm{coh}_{\text {left }}=-\mathrm{coh}_{\text {right }}$. The excitatory input for each pathway is set the same way as it is above, however now the maximum conductance of these input synapses $g_{\text {sen }}$ is one free parameter.

The second component of the network is a decision network. This network is a two-pool rate mode ${ }^{38}$, using the parameter set therein with no recurrent AMPAR current. The pool representing choice 1 receives input from a subset of the left-red neuron pool in the mixed-sensory network. Sensory neurons are sorted according to their gating selectivity, and only the top $P_{\text {project }}$ fraction of these sensory neurons project to the decision networks. $P_{\text {project }}$ is also a free parameter. The right-green pool projects to the choice 2 pool. The other two pools do not project to the decision network because only the left-red and the right-green pools have congruent preferences for choice 1 and choice 2, respectively, based on the how colour and motion evidence are defined. To fit experimental behavioural choice data efficiently, we further approximated the decision network with a decision function. We assumed that the probability of selecting choice $1\left(P_{1}\right)$ is determined by the difference $\Delta I_{\mathrm{dec}}(\mathrm{pA})$ between the input currents to the two choice pools. We fitted this function by simulating the decision network with mean input current $15.6 \mathrm{pA}$ to both pools, yielding

$$
P_{1}=\left[1+\exp \left(\frac{-\Delta I_{\mathrm{dec}}}{\sigma}\right)\right]^{-1}
$$

with $\sigma=0.99 \mathrm{pA}$. The second free parameter of the model is the projection strength $J_{\mathrm{dec}}$ of the mixed-sensory input, such that $\Delta I_{\mathrm{dec}}=J_{\mathrm{dec}}\left(r_{\text {left,red }}-r_{\text {right,green }}\right) . r_{\text {left,red }}$ is the average firing rate of the left/red-preferring pool.
The three free parameters $g_{\mathrm{sen}}, P_{\text {project }}$ and $J_{\mathrm{dec}}$ are fitted to behavioural data of each monkey in ref. 28. The fit parameter values are $g_{\text {sen }}=1.21 \mathrm{nS}, P_{\text {project }}=0.36$ and $J_{\mathrm{dec}}=15.0 \mathrm{pA} \mathrm{Hz}^{-1}$ for monkey $\mathrm{F}$, and are $g_{\text {sen }}=1.80 \mathrm{nS}, P_{\text {project }}=0.083$ and $J_{\mathrm{dec}}=4.37 \mathrm{pA} \mathrm{Hz}^{-1}$ for monkey A. Importantly, the data used to fit the model are far from being sufficient. Also, our circuit model is simplistic. Therefore, these fitted parameter values do not reflect our estimates of these quantities in the brain Rather, these fittings demonstrate that the proposed circuit architecture can potentially capture behavioural performance. As shown in Supplementary Fig. 8, if neural gating is strongly degraded, then no set of these fit parameters can capture behavioural performance.

Model fitting in general. Model parameters are fitted to experimental or simulation data in various contexts. These fitted models include the rate pyramidal neuron, the calcium-based plasticity model and the context-dependent decisionmaking network. In all these cases, parameters are chosen to minimize the squarederror between the model and data using sequential least squares programming method from the SciPy library (scipy.optimize.minimize, with method 'sequential least squares programming').

Data availability. Code that supports the findings of this study is publicly available on Github: https://github.com/gyyang/DisinhibitoryCircuit2016. All other data can be found within this article and its Supplementary Material.

\section{References}

1. Markram, H. et al. Interneurons of the neocortical inhibitory system. Nat. Rev. Neurosci. 5, 793-807 (2004).

2. Jiang, X. et al. Principles of connectivity among morphologically defined cell types in adult neocortex. Science 350, aac9462 (2015).

3. Urban-Ciecko, J. \& Barth, A. L. Somatostatin-expressing neurons in cortical networks. Nat. Rev. Neurosci. 17, 401-409 (2016).

4. Chiu, C. Q. et al. Compartmentalization of GABAergic inhibition by dendritic spines. Science 340, 759-762 (2013).

5. Pfeffer, C. K., Xue, M., He, M., Huang, Z. J. \& Scanziani, M. Inhibition of inhibition in visual cortex: the logic of connections between molecularly distinct interneurons. Nat. Neurosci. 16, 1068-1076 (2013).

6. Lee, S., Kruglikov, I., Huang, Z. J., Fishell, G. \& Rudy, B. A disinhibitory circuit mediates motor integration in the somatosensory cortex. Nat. Neurosci. 16, $1662-1670$ (2013).

7. Zhang, S. et al. Long-range and local circuits for top-down modulation of visual cortex processing. Science 345, 660-665 (2014).

8. Fu, Y. et al. A cortical circuit for gain control by behavioral state. Cell 156, 1139-1152 (2014).

9. Wang, X.-J., Tegner, J., Constantinidis, C. \& Goldman-Rakic, P. S. Division of labor among distinct subtypes of inhibitory neurons in a cortical microcircuit of working memory. Proc. Natl Acad. Sci. USA 101, 1368-1373 (2004).

10. Kepecs, A. \& Fishell, G. Interneuron cell types are fit to function. Nature 505, 318-326 (2014).

11. Sridharan, D. \& Knudsen, E. I. Selective disinhibition: a unified neural mechanism for predictive and post hoc attentional selection. Vision Res. 116, 194-209 (2015).

12. Pinto, L. \& Dan, Y. Cell-type-specific activity in prefrontal cortex during goaldirected behavior. Neuron 87, 437-450 (2015).

13. Kvitsiani, D. et al. Distinct behavioural and network correlates of two interneuron types in prefrontal cortex. Nature 498, 363-366 (2013).

14. Pi, H.-J. et al. Cortical interneurons that specialize in disinhibitory control. Nature 503, 521-524 (2013).

15. Gentet, L. J. et al. Unique functional properties of somatostatin-expressing GABAergic neurons in mouse barrel cortex. Nat. Neurosci. 15, 607-612 (2012).

16. Ma, W.-P. et al. Visual representations by cortical somatostatin inhibitory neurons-selective but with weak and delayed responses. J. Neurosci. 30, 14371-14379 (2010).

17. Adler, A. \& Gan, W.-B. Program No. 357.10. 2015 Neuroscience Meeting Planner (Society for Neuroscience, 2015).

18. Akam, T. \& Kullmann, D. M. Oscillations and filtering networks support flexible routing of information. Neuron 67, 308-320 (2010).

19. Vogels, T. P. \& Abbott, L. F. Gating multiple signals through detailed balance of excitation and inhibition in spiking networks. Nat. Neurosci. 12, 483-491 (2009).

20. Poirazi, I. P., Brannon, T. \& Mel, B. W. Pyramidal neuron as two-layer neural network. Neuron 37, 989-999 (2003).

21. Major, G., Larkum, M. E. \& Schiller, J. Active properties of neocortical pyramidal neuron dendrites. Annu. Rev. Neurosci. 36, 1-24 (2013).

22. Petreanu, L., Mao, T., Sternson, S. M. \& Svoboda, K. The subcellular organization of neocortical excitatory connections. Nature 457, 1142-1145 (2009).

23. Druckmann, S. et al. Structured synaptic connectivity between hippocampal regions. Neuron 81, 629-640 (2014). 
24. Yang, G. et al. Sleep promotes branch-specific formation of dendritic spines after learning. Science 344, 1173-1178 (2014).

25. Kastellakis, G., Cai, D. J., Mednick, S. C., Silva, A. J. \& Poirazi, P. Synaptic clustering within dendrites: An emerging theory of memory formation. Prog. Neurobiol. 126, 19-35 (2015).

26. Urban-Ciecko, J., Fanselow, E. E. \& Barth, A. L. Neocortical somatostatin neurons reversibly silence excitatory transmission via GABAb receptors. Curr. Biol. 25, 722-731 (2015).

27. Fino, E. \& Yuste, R. Dense inhibitory connectivity in neocortex. Neuron 69, 1188-1203 (2011).

28. Mante, V., Sussillo, D., Shenoy, K. V. \& Newsome, W. T. Context-dependent computation by recurrent dynamics in prefrontal cortex. Nature 503, 78-84 (2013).

29. Karnani, M. M., Agetsuma, M. \& Yuste, R. A blanket of inhibition: functional inferences from dense inhibitory connectivity. Curr. Opin. Neurobiol. 26, 96-102 (2014)

30. Schiller, J., Major, G., Koester, H. J. \& Schiller, Y. NMDA spikes in basal dendrites of cortical pyramidal neurons. Nature 404, 285-289 (2000).

31. Jadi, M., Polsky, A., Schiller, J. \& Mel, B. W. Location-dependent effects of inhibition on local spiking in pyramidal neuron dendrites. PLoS Comput. Biol. 8, e1002550 (2012).

32. Silberberg, G. \& Markram, H. Disynaptic inhibition between neocortical pyramidal cells mediated by Martinotti cells. Neuron 53, 735-746 (2007).

33. Bayraktar, T., Welker, E., Freund, T. F., Zilles, K. \& Staiger, J. F. Neurons immunoreactive for va-soactive intestinal polypeptide in the rat primary somatosensory cortex: morphology and spatial relationship to barrel-related columns. J. Comp. Neurol. 420, 291-304 (2000).

34. Fu, Y., Kaneko, M., Tang, Y., Alvarez-Buylla, A. \& Stryker, M. P. A cortical disinhibitory circuit for enhancing adult plasticity. Elife 4, e05558 (2014).

35. Bar-Ilan, L., Gidon, A. \& Segev, I. The role of dendritic inhibition in shaping the plasticity of excitatory synapses. Front. Neural Circuits 6, 118 (2012).

36. Graupner, M. \& Brunel, N. Calcium-based plasticity model explains sensitivity of synaptic changes to spike pattern, rate, and dendritic location. Proc. Natl Acad. Sci. USA 109, 3991-3996 (2012).

37. Nevian, T. \& Sakmann, B. Spine $\mathrm{Ca} 2+$ signaling in spike-timing-dependent plasticity. J. Neurosci. 26, 11001-11013 (2006).

38. Wong, K.-F. \& Wang, X.-J. A recurrent network mechanism of time integration in perceptual decisions. J. Neurosci. 26, 1314-1328 (2006)

39. Lovett-Barron, M. et al. Regulation of neuronal input transformations by tunable dendritic inhibition. Nat. Neurosci. 15, 423-430 S1-3 (2012).

40. Chen, S. X., Kim, A. N., Peters, A. J. \& Komiyama, T. Subtype-specific plasticity of inhibitory circuits in motor cortex during motor learning. Nat Neurosci. 18, 1109-1115 (2015).

41. Vogels, T. P., Sprekeler, H., Zenke, F., Clopath, C. \& Gerstner, W. Inhibitory plasticity balances excitation and inhibition in sensory pathways and memory networks. Science 334, 1569-1573 (2011).

42. Xue, M., Atallah, B. V. \& Scanziani, M. Equalizing excitation-inhibition ratios across visual cortical neurons. Nature 511, 596-600 (2014).

43. Koch, C., Poggio, T. \& Torre, V. Retinal ganglion cells: a functional interpretation of dendritic morphology. Philos. Trans. R. Soc. Lond. B Biol. Sci. 298, 227-263 (1982).

44. Kleindienst, T., Winnubst, J., Roth-Alpermann, C., Bonhoeffer, T. \& Lohmann, C. Activity-dependent clustering of functional synaptic inputs on developing hippocampal dendrites. Neuron 72, 1012-1024 (2011).

45. Fu, M., Yu, X., Lu, J. \& Zuo, Y. Repetitive motor learning induces coordinated formation of clustered dendritic spines in vivo. Nature 483, 92-95 (2012).

46. Cichon, J. \& Gan, W.-B. Branch-specific dendritic $\mathrm{Ca}(2+)$ spikes cause persistent synaptic plasticity. Nature 520, 180-185 (2015).

47. Smith, S. L., Smith, I. T., Branco, T. \& Hausser, M. Dendritic spikes enhance stimulus selectivity in cortical neurons in vivo. Nature 503, 115-120 (2013).

48. Abbott, L. 23 Problems in Systems Neuroscience 423-431 (Oxford Univ. Press (2006).

49. Olshausen, B. A., Anderson, C. H. \& Van Essen, D. C. A neurobiological model of visual attention and invariant pattern recognition based on dynamic routing of information. J. Neurosci. 13, 4700-4719 (1993).

50. Kepecs, A. \& Raghavachari, S. Gating information by two-state membrane potential fluctuations. J. Neurophysiol. 97, 3015-3023 (2007).

51. Zylberberg, A., Fernandez Slezak, D., Roelfsema, P. R., Dehaene, S. \& Sigman, M. The brain's router: a cortical network model of serial processing in the primate brain. PLoS Comput. Biol. 6, e1000765 (2010).

52. Branco, T., Clark, B. A. \& Hausser, M. Dendritic discrimination of temporal input sequences in cortical neurons. Science 329, 1671-1675 (2010).

53. Hines, M. L. \& Carnevale, N. T. The NEURON simulation environment. Neural. Comput. 9, 1179-1209 (1997).
54. Murphy, B. K. \& Miller, K. D. Multiplicative gain changes are induced by excitation or inhibition alone. J. Neurosci. 23, 10040-10051 (2003).

55. Nevian, T., Larkum, M. E., Polsky, A. \& Schiller, J. Properties of basal dendrites of layer 5 pyramidal neurons: a direct patch-clamp recording study. Nat. Neurosci. 10, 206-214 (2007).

56. Larkum, M. E., Senn, W. \& Luscher, H.-R. Top-down dendritic input increases the gain of layer 5 pyramidal neurons. Cereb. Cortex. 14, 1059-1070 (2004).

57. Rudolph, M. \& Destexhe, A. A fast-conducting, stochastic integrative mode for neocortical neurons in vivo. J. Neurosci. 23, 2466-2476 (2003).

58. Goodman, D. \& Brette, R. Brian: a simulator for spiking neural networks in python. Front. Neuroinform. 2, 5 (2008).

59. Marlin, J. J. \& Carter, A. G. GABA-A receptor inhibition of local calcium signaling in spines and dendrites. J. Neurosci. 34, 15898-15911 (2014).

60. Ali, A. B. \& Thomson, A. M. Synaptic alpha 5 subunit-containing GABAAreceptors mediate IPSPs elicited by dendrite-preferring cells in rat neocortex. Cereb. Cortex. 18, 1260-1271 (2008).

61. Shoemaker, P. A. Neural bistability and amplification mediated by NMDA receptors: analysis of stationary equations. Neurocomputing 74, 3058-3071 (2011).

62. Ascher, P. \& Nowak, L. The role of divalent cations in the N-methyl-Daspartate responses of mouse central neurones in culture. J. Physiol. 399, 247-266 (1988).

63. Ishikawa, T., Sahara, Y. \& Takahashi, T. A single packet of transmitter does not saturate post-synaptic glutamate receptors. Neuron 34, 613-621 (2002).

64. Popescu, G., Robert, A., Howe, J. R. \& Auerbach, A. Reaction mechanism determines NMDA receptor response to repetitive stimulation. Nature 430, 790-793 (2004)

65. Lu, J.-T., Li, C.-Y., Zhao, J.-P., Poo, M.-M. \& Zhang, X.-H. Spike-timingdependent plasticity of neocortical excitatory synapses on inhibitory interneurons depends on target cell type. J. Neurosci. 27, 9711-9720 (2007)

66. Lee, S., Hjerling-Leffler, J., Zagha, E., Fishell, G. \& Rudy, B. The largest group of superficial neocortical GABAergic interneurons expresses ionotropic serotonin receptors. J. Neurosci. 30, 16796-16808 (2010).

67. Larkman, A. U. Dendritic morphology of pyramidal neurones of the visual cortex of the rat:III. Spine distributions. J. Comp. Neurol. 306, 332-343 (1991).

68. Higgins, D., Graupner, M. \& Brunel, N. Memory maintenance in synapses with calcium-based plasticity in the presence of background activity. PLoS Comput. Biol. 10, e1003834 (2014).

69. Britten, K. H., Shadlen, M. N., Newsome, W. T. \& Movshon, J. A. Responses of neurons in macaque MT to stochastic motion signals. Vis. Neurosci. 10, 1157-1169 (1993).

\section{Acknowledgements}

We thank Michael Higley, Alex Kwan, Jorge Jaramillo and Francis Song for comments on an earlier version of the manuscript. Funding was provided by National Institute of Health grant R01MH062349 and Office of Naval Research grant N00014-13-1-0297 (X.-J.W.).

\section{Author contributions}

G.R.Y., J.D.M. and X.-J.W. designed the research, discussed regularly throughout the project and wrote the manuscript. G.R.Y. performed the research.

\section{Additional information}

Supplementary Information accompanies this paper at http://www.nature.com/ naturecommunications

Competing financial interests: The authors declare no competing financial interests

Reprints and permission information is available online at http://npg.nature.com/ reprintsandpermissions/

How to cite this article: Yang, G.R. et al. A dendritic disinhibitory circuit mechanism for pathway-specific gating. Nat. Commun. 7:12815 doi: 10.1038/ncomms12815 (2016).

This work is licensed under a Creative Commons Attribution 4.0 International License. The images or other third party material in this article are included in the article's Creative Commons license, unless indicated otherwise in the credit line; if the material is not included under the Creative Commons license, users will need to obtain permission from the license holder to reproduce the material To view a copy of this license, visit http://creativecommons.org/licenses/by/4.0/ 